\title{
Genetic and Pathogenic Relatedness of Pseudoperonospora cubensis and P. humuli
}

\author{
Melanie N. Mitchell, Cynthia M. Ocamb, Niklaus J. Grünwald, Leah E. Mancino, and David H. Gent
}

First, second, and fourth authors: Department of Botany and Plant Pathology, third author: United States Department of AgricultureAgricultural Research Service (USDA-ARS), Horticultural Crops Research Unit; and fifth author: USDA-ARS, Forage Seed and Cereal Research Unit, and Department of Botany and Plant Pathology, Oregon State University, Corvallis.

Accepted for publication 6 March 2011.

\begin{abstract}
Mitchell, M. N., Ocamb, C. M., Grünwald, N. J., Mancino, L. E., and Gent, D. H. 2011. Genetic and pathogenic relatedness of Pseudoperonospora cubensis and P. humuli. Phytopathology 101:805-818.

The most economically important plant pathogens in the genus Pseudoperonospora (family Peronosporaceae) are Pseudoperonospora cubensis and $P$. humuli, causal agents of downy mildew on cucurbits and hop, respectively. Recently, $P$. humuli was reduced to a taxonomic synonym of $P$. cubensis based on internal transcribed spacer (ITS) sequence data and morphological characteristics. Nomenclature has many practical implications for pathogen identification and regulatory considerations; therefore, further clarification of the genetic and pathogenic relatedness of these organisms is needed. Phylogenetic analyses were conducted considering two nuclear and three mitochondrial loci for 21 isolates of P. cubensis and 14 isolates of $P$. humuli, and all published ITS sequences of the pathogens in GenBank. There was a consistent separa-

in nuclear, mitochondrial, and ITS phylogenetic analyses, with the exception of isolates of $P$. humuli from Humulus japonicus from Korea. The $P$. cubensis isolates appeared to contain the $P$. humuli cluster, which may indicate that $P$. humuli descended from $P$. cubensis. Host-specificity experiments were conducted with two reportedly universally susceptible hosts of $P$. cubensis and two hop cultivars highly susceptible to $P$. humuli. $P$. cubensis consistently infected the hop cultivars at very low rates, and sporangiophores invariably emerged from necrotic or chlorotic hypersensitive-like lesions. Only a single sporangiophore of $P$. humuli was observed on a cucurbit plant during the course of the studies. Together, molecular data and host specificity indicate that there are biologically relevant characteristics that differentiate $P$. cubensis and $P$. humuli that may be obfuscated if $P$. humuli were reduced to a taxonomic synonym of $P$. cubensis. Thus, we recommend retaining the two species names $P$. cubensis and $P$. humuli until the species boundaries can be resolved unambiguously.
\end{abstract} tion of the majority of the $P$. humuli isolates and the $P$. cubensis isolates
Downy mildews are members of the family Peronosporaceae (Oomycota, Oomycetes, Peronosporales), which comprise one of the largest groups of fungi-like organisms that parasitize flowering plants (42). Within the family Peronosporaceae are six species of Pseudoperonospora Rostovzev. Economically important species of the genus include Pseudoperonospora cubensis (Berk. \& M. A. Curtis) Rostovzev (the type species) and P. humuli (Miyabe \& Takah.) G. W. Wilson. P. cubensis, causal agent of cucurbit downy mildew, has been found on at least 49 wild and cultivated species of cucurbits in 70 countries $(6,7,41)$. Cucurbit downy mildew affects 9 of the 12 cultivated cucurbit species $(6,7,60)$. $P$. cubensis infects plants in both the field and protected cultivation in tropical areas around the world, as well as in some semiarid and temperate regions. $P$. humuli, the causal agent of hop downy mildew, is found mainly in hop-production regions throughout the northern hemisphere (37). The commercial host of P. humuli is the hop plant (Humulus lupulus L.), a dioecious plant in the family Cannabaceae.

The downy mildews have been a difficult group for determining what defines a species. Morphometric techniques, which include measurements of the size, shape, color, and so on of anatomical features, have been commonly used to distinguish and identify a species in many areas of biology. These techniques can be

Corresponding author: D. H. Gent; E-mail address: gentd@onid.orst.edu

doi:10.1094/PHYTO-10-10-0270

This article is in the public domain and not copyrightable. It may be freely reprinted with customary crediting of the source. The American Phytopathological Society, 2011 uninformative for downy mildew pathogens $(11,18,44)$. This is mainly due to the few visible characteristics, because nearly all but the reproductive structures are within plant tissue and many characteristics can vary widely depending on the host matrix and the environmental conditions $(22,51)$. Because downy mildew pathogens are obligate biotrophs, the traditional biological species concept based on mating compatibility is difficult to test experimentally.

To overcome these problems with morphology, Gäumann (13) proposed a biological species concept for downy mildews based on host specialization (22,59). Gäumann (13) proposed that a species of the family Peronosporaceae would be specific to a single host genus or even species. However, few cross-infection experiments were performed to confirm his strict assumption about host specificity, sometimes leading to artificial splitting of some species with polyphagous host ranges $(13,22,59)$. This concept is also problematic when multiple species of downy mildew are parasitic on a single host species $(59,64)$.

The ecophysiophenetic concept is essentially the intersection between the biological and morphometric species concepts (22). In this concept, the criterion for the delimitation of a species is dependent upon host specificity (to host genus or family) and morphology of the conidiophores and conidia or sporangiophores and sporangia (22). Although this concept appears as the middle ground, it suffers from many of the same problems as the morphometric and biological species concepts.

In response to these problems, and with the advent of molecular techniques, phylogenetic analyses of the downy mildews have been used to infer reproductive isolation and delineate species that are monophyletic. According to Hall (22), "species are 
defined as clusters of organisms diagnosably different from other clusters and within which there are parental networks of ancestry and descent." The determination of whether a cluster, or clade, is diagnostically different from other clusters relies on statistical probabilities and models to reconstruct phylogenies. However, how different a clade has to be to represent a species is not clear and is influenced by the locus, number of loci, and characteristics used as well as the sample size and how well the sample represents the species as a whole. For example, in the Oomycete genus Phytophthora, Phytophthora infestans, $P$. mirabilis, and $P$. ipomoea are mainly distinguished through host range studies while they are almost identical for internal transcribed spacer (ITS) sequences $(12,19)$.

Pseudoperonospora cubensis and $P$. humuli have been found to be sister taxa nested within other Pseudoperonospora spp. in phylogenetic analyses of molecular data $(6,45,62,63)$. Choi et al. (6) suggested that $P$. cubensis and $P$. humuli were synonymous based on overlapping dimensions of certain morphometric characteristics and ITS sequence data of nine isolates of each pathogen. Runge et al. (49) challenged the proposed synonymy based on a multigenetic analysis of a larger collection of $P$. cubensis isolates from east Asia, Europe, and the Americas. They included a modest sampling of $P$. humuli (six isolates) in their analysis, none of which were from North America.

P. cubensis can exhibit different morphology on different hosts and in different environmental conditions $(6,41,51)$. Thus, the slight differences between the morphology of the downy mildew pathogens could be attributed to the same phenomenon if the two really are the same species. It is interesting to note that the ranges for sporangiophore length and trunk width and sporangial dimensions given in Choi et al. (6) are broader than those reported by Palti (40) and Miyabe and Takahashi (36).

In the genera Pythium and Phytophthora, several species have identical or nearly identical ITS sequences, indicating that this may not be the ideal locus for distinguishing oomycete species $(27,30,33)$. Analysis of multiple genetic regions is clearly required to distinguish closely related taxa (2) such as $P$. cubensis and $P$. humuli. Currently, a multigenic, phylogenetic analysis of $P$. cubensis and $P$. humuli is absent and the conclusions based solely on ITS analysis might not be adequate.

Previous studies $(6,49)$ did not report on host specificity among isolates of $P$. cubensis and $P$. humuli, which is a critical characteristic for regulatory consideration of downy mildews. $P$. humuli may infect Urtica, Cannabis, and Celtis spp. at a low level following artificial inoculation $(24,52)$. There is no record of $P$. cubensis successfully infecting wild or cultivated hop, or of infection of cucurbits by $P$. humuli. Hoerner (24) reported that "all attempts to infect available hosts of Pseudoperonospora cubensis [with $P$. humuli] ... were unsuccessful." The proposed reduction from two pathogenic species into one has implications for pathogen identification, disease management, and regulatory considerations; therefore, a critical evaluation of this proposal is required. To this end, our objectives were to examine the relationship between $P$. cubensis and P. humuli in terms of (i) host ranges and (ii) a multigenic, phylogenetic analysis based on a diverse collection of isolates from both taxa.

\section{MATERIALS AND METHODS}

Plant material. Plants of the downy-mildew-susceptible hop 'Nugget' (23) and 'Pacific Gem' were propagated from softwood cuttings and maintained in a greenhouse free of downy mildews. Plants of the susceptible cucumber 'Straight 8' (54) and cantaloupe 'Ananes Yokneam' $(29,60)$ were grown from seed in the same greenhouse. The greenhouse was maintained at 20 to $25^{\circ} \mathrm{C}$ with a 14-h photoperiod. Hop plants were grown in Sunshine Mix number 1 (SunGro Horticulture, Bellevue, WA) in $440-\mathrm{cm}^{3}$ pots for $\approx 14$ days for use in host-specificity experiments (described below). Some hop plants were repotted into $648-\mathrm{cm}^{3}$ pots for an additional 14 days for maintenance of $P$. humuli isolates. Cucurbit plants were grown in the same soil in $440-\mathrm{cm}^{3}$ pots for $\approx 4$ to 6 weeks. Plants were watered regularly and supplied with Champion 17-17-17 $\left(\mathrm{N}-\mathrm{P}_{2} \mathrm{O}_{5}-\mathrm{K}_{2} \mathrm{O}\right)$ fertilizer with micronutrients (McConkey's, Portland, OR) at each irrigation to promote succulent growth.

Isolates. Isolates of P. humuli were collected during 2006 to 2009 from Oregon (eight isolates) and Washington (three isolates) (Table 1). Monosporangial field isolates were attained from symptomatic hop shoots infected with $P$. humuli as described by Gent et al. (15). Isolates were maintained and increased for DNA extraction from sporangia on Nugget hop using a droplet inoculation procedure on detached leaves (2006 to 2008) as described by Gent et al. (15) or a spray inoculation procedure for whole plants (during 2009). Additionally, two DNA samples of $P$. humuli were received from the Czech Republic and one DNA sample was received from Korea (Table 1).

For the spray inoculation, sporangia were dislodged from sporulating leaves using a pressurized narrow stream of sterile 18ohm water (MilliQ water; Millipore, Billerica, MA) produced by a Preval Complete Spray Unit (Precision Valve Corporation, Yonkers, NY). The inoculum for maintenance of cultures was adjusted to at least $5 \times 10^{4}$ sporangia $\mathrm{ml}^{-1}$ using a hemacytometer and was sprayed to runoff onto the underside of leaves of Nugget hop. One inoculated plant (in a $440-\mathrm{cm}^{3}$ pot) was placed into a prepared 90-ounce $\left(2,661.6-\mathrm{cm}^{3}\right)$ plastic container (Pasta Keeper, 29.21 by 11.43 by $11.43 \mathrm{~cm}$; Snapware, Mira Loma, CA) or three inoculated plants (in a $648-\mathrm{cm}^{3}$ pot) were placed into a prepared 2.5-gallon $\left(11,012.2-\mathrm{cm}^{3}\right)$ plastic barrel container ( 35.56 by 20.96 by $20.96 \mathrm{~cm}$; Snapware). The containers were prepared by having sterile 18 -ohm water sprayed onto the inside walls and inside of the lid to increase humidity. A moistened paper towel folded twice into a square was placed at the bottom of the container to aid in increasing the humidity. The inoculated plants were kept overnight in the containers with the lids closed. The following morning, the plants were removed from the containers and allowed to air dry for $24 \mathrm{~h}$ before being replaced into the dried containers and then placed into a growth chamber for 6 to 8 days. Isolates were maintained on host plants at $20^{\circ} \mathrm{C}$ with a 12 -h light photoperiod provided by fluorescent lights $\left(\approx 300 \mu \mathrm{mol} / \mathrm{m}^{2} / \mathrm{s}\right)$. After 6 days of incubation, sporulation was induced by spraying the inside walls and lid of the container with deionized water and closing the container overnight.

$P$. cubensis isolates from the United States were received from other researchers during 2005 to 2009 and included isolates from California (6), North Carolina (9), New Jersey (1), Oregon (2), Michigan (2), and Ohio (1) (Table 1). Some of these isolates were previously characterized to pathotype (10) and were found to be unique pathotypes based on their reaction on the differential hosts described by Lebeda and Widrlechner (29). Isolates from infected cucurbits leaves were maintained on Straight 8 cucumber using a spray inoculation procedure on whole plants, the same as for $P$. humuli, except that the inoculum was adjusted to $\approx 6.5 \times 10^{3}$ spores $\mathrm{ml}^{-1}$. Isolates of $P$. cubensis were not monosporangial due to difficulties in attaining a monosporangial isolate of the pathogen. After inoculation, cucumber plants were put into prepared closed plastic containers (either one plant in a Pasta Keeper or two to three plants in a barrel container) as described previously and were put into a dark, humid chamber (at least $98 \%$ humidity, $21^{\circ} \mathrm{C}$ ) for $24 \mathrm{~h}$. After $24 \mathrm{~h}$, the plants were removed from the plastic containers and placed into trays holding at least 1 liter of deionized water in a growth chamber at 21 and $18^{\circ} \mathrm{C}$ (day and night, respectively) with a 12 -h photoperiod. To induce sporulation after 6 days, the inside walls and lid of the container were sprayed with deionized water and the container was closed and placed into a dark humid chamber overnight. Each isolate was maintained in a separate chamber to avoid cross-contami- 
nation. Additionally, herbarium samples of $P$. cubensis and $P$. celtidis were received from South Korea (Table 1).

DNA extraction. DNA was extracted from sporangial suspensions using a cetyl trimethylammonium bromide (CTAB) procedure modified from Chee et al. (5) or a MoBio Ultra Clean Soil DNA Isolation Kit (MoBio Laboratories, Carlsbad, CA) according to the manufacturer's instructions, with modifications. The modified CTAB extraction was conducted as follows. Sporangial suspensions were centrifuged at $10,600 \times g$ for $3 \mathrm{~min}$ and then resuspended in $100 \mu \mathrm{l}$ of $1 \times$ Tris-EDTA (TE). Polyvinyl pyrrolidone (PVP) $(0.021 \mathrm{~g})$, CTAB extraction buffer $(900 \mu \mathrm{l} ; 100 \mathrm{mM}$ Tris- $\mathrm{HCl}$ [pH 7.5], 1.4 M NaCl, $20 \mathrm{mM}$ EDTA, and $5.49 \mathrm{mM}$ CTAB), and $100 \mu \mathrm{l}$ of the sporangial suspension in TE were added to a FastPrep Lysing Matrix A Tube (MP Biomedicals, Solon, $\mathrm{OH}$ ) and put into a FastPrep instrument (Bio 101, Vista, $\mathrm{CA}$ ), which was run five times at level 6 for $45 \mathrm{~s}$; tubes were placed on ice for $2 \mathrm{~min}$ between each run. Then, $750 \mu \mathrm{l}$ of the solution was transferred to a $1.5-\mathrm{ml}$ microfuge tube to which $7.5 \mu \mathrm{l}$ of $\beta$-mercaptoethanol, $22.5 \mu \mathrm{l}$ of proteinase $\mathrm{K}$ at $20 \mathrm{mg} \mathrm{ml}^{-1}$, and $7.5 \mu \mathrm{l}$ of RNase $\mathrm{A}$ at $10 \mathrm{mg} / \mathrm{ml}$ was added, followed by incubation at $65^{\circ} \mathrm{C}$ for $30 \mathrm{~min}$. After the solution was mixed with $750 \mu \mathrm{l}$ of $24: 1$ chloroform/isoamyl alcohol, it was centrifuged at $10,600 \times g$ for $10 \mathrm{~min}$. Up to $650 \mu \mathrm{l}$ of the aqueous phase was then transferred to a $1.5-\mathrm{ml}$ microfuge tube and the chloroform/ isoamyl alcohol addition and centrifugation steps were repeated. Up to $500 \mu \mathrm{l}$ of the aqueous phase was transferred to a $1.5-\mathrm{ml}$ microfuge tube and nucleic acids were precipitated by addition of an equal volume of cold isopropanol. The DNA was pelleted by centrifugation at $10,600 \times g$ for $20 \mathrm{~min}$ at $4^{\circ} \mathrm{C}$. The pellet was subsequently rinsed twice with $70 \%$ ethanol, dried in a fume hood on a hot plate at $35^{\circ} \mathrm{C}$, and then resuspended in $50 \mu \mathrm{l}$ of $10 \mathrm{mM}$ Tris- $\mathrm{HCl}, \mathrm{pH} 7.5$, and stored at $-20^{\circ} \mathrm{C}$.

The modifications to the MoBio Ultra Clean Soil DNA Isolation Kit were as follows. To solution S1, $0.140 \mathrm{~g}$ of PVP was added to improve the fidelity of polymerase chain reaction (PCR) amplification following extraction (14). After the addition of S1, $200 \mu \mathrm{l}$ of inhibitor removal solution (IRS) (MoBio Laboratories) was added to the tube with the bead lysis solution. For higher recovery of DNA, the tube with the bead lysis solution, sporangial suspension, and solutions S1 and IRS was boiled for 2 min before

TABLE 1. Identity and origin of organisms used in this study, including notes on isolates used in different host-specificity studies

\begin{tabular}{|c|c|c|c|c|c|c|c|}
\hline \multirow[b]{2}{*}{ Organism } & \multirow[b]{2}{*}{ Isolate } & \multirow[b]{2}{*}{ Host } & \multirow[b]{2}{*}{ Collection location } & \multirow[b]{2}{*}{ Year } & \multicolumn{3}{|c|}{ GenBank accession number ${ }^{a}$} \\
\hline & & & & & ITS & $\beta$-Tubulin & $\operatorname{cox}$ Cluster \\
\hline Pseudoperonospora cubensis & CDM-237 & Citrullus lanatus & United States, New Jersey & 2007 & JF304653 & JF304687 & JF414537 \\
\hline P. cubensis & CDM-241 & Cucurbita sp. & United States, North Carolina & 2007 & JF304654 & JF304688 & JF414538 \\
\hline P. cubensis & CDM-246 & Momordica charantia & United States, North Carolina & 2007 & JF304655 & JF304689 & JF414539 \\
\hline P. cubensis & CDM-247 & Cucurbita sp. & United States, New Jersey & 2007 & JF304656 & JF304690 & JF414540 \\
\hline P. cubensis & CDM-248 & Cucurbita pepo & United States, North Carolina & Unknown & JF304657 & JF304691 & JF414541 \\
\hline P. cubensis $^{\mathrm{b}}$ & CDM-251 & Cucumis sativus & United States, Michigan & 2007 & JF304658 & JF304692 & JF414542 \\
\hline P. cubensis $^{\mathrm{b}, \mathrm{c}}$ & CDM-252 & C. sativus & United States, Ohio & 2007 & JF304659 & JF304693 & JF414543 \\
\hline P. cubensis $^{\mathrm{b}}$ & CDM-253 & C. sativus & United States, North Carolina & 2007 & JF304660 & JF304694 & JF414544 \\
\hline P. cubensis $^{\mathrm{c}}$ & CDM-254 & C. sativus & United States, North Carolina & 2006 & JF304661 & JF304695 & JF414545 \\
\hline P. cubensis $^{\mathrm{b}, \mathrm{c}}$ & CDM-255 & C. sativus & United States, Michigan & 2005 & JF304662 & JF304696 & JF414546 \\
\hline P. cubensis & CDM-266 & C. sativus & United States, California & 2009 & JF304663 & JF304697 & JF414547 \\
\hline P. cubensis & CDM-268 & C. sativus & United States, California & 2009 & JF304664 & JF304698 & JF414548 \\
\hline P. cubensis & CDM-269 & C. sativus & United States, California & 2009 & JF304665 & JF304699 & JF414549 \\
\hline P. cubensis & CDM-272 & C. sativus & United States, California & 2009 & JF304666 & JF304700 & JF414550 \\
\hline P. cubensis & CDM-273 & C. sativus & United States, California & 2009 & JF304667 & JF304701 & JF414551 \\
\hline P. cubensis & CDM-274 & C. sativus & United States, California & 2009 & JF304668 & JF304702 & JF414552 \\
\hline P. cubensis $^{\mathrm{c}}$ & CDM-275 & Cucurbita реро & United States, North Carolina & 2005 & JF304669 & JF304703 & JF414553 \\
\hline P. cubensis $^{\mathrm{c}}$ & CDM-276 & С. реро & United States, North Carolina & 2006 & JF304670 & JF304704 & JF414554 \\
\hline P. cubensis $^{\mathrm{c}}$ & CDM-277 & C. moschata & United States, North Carolina & 2008 & JF304671 & JF304705 & JF414555 \\
\hline P. cubensis & CDM-278 & Cucumis sativus & United States, Oregon & 2009 & JF304672 & JF304706 & JF414556 \\
\hline P. cubensis & CDM-279 & C. sativus & United States, Oregon & 2009 & JF304673 & JF304707 & JF414557 \\
\hline P. cubensis $^{\mathrm{c}}$ & CDM-282 & C. melo & United States, South Carolina & 2010 & $\ldots$ & $\ldots$ & $\ldots$ \\
\hline P. humuli & HDM-094 & Humulus lupulus & United States, Washington & 2006 & JF304674 & JF304708 & JF414558 \\
\hline P. humuli & HDM-103 & H. lupulus & United States, Washington & 2006 & JF304675 & JF304709 & JF414559 \\
\hline P. humuli & HDM-110 & H. lupulus & United States, Oregon & 2006 & JF304676 & JF304710 & JF414560 \\
\hline P. humuli & HDM-140 & H. lupulus & United States, Oregon & 2006 & JF304677 & JF304711 & JF414561 \\
\hline P. humuli & HDM-158 & H. lupulus & United States, Oregon & 2007 & JF304678 & JF304712 & JF414562 \\
\hline P. humuli & HDM-170 & H. lupulus & United States, Oregon & 2007 & JF304679 & JF304713 & JF414563 \\
\hline P. humuli & HDM-171 & H. lupulus & United States, Oregon & 2007 & JF304680 & JF304714 & JF414564 \\
\hline P. humuli ${ }^{\mathrm{d}, \mathrm{e}}$ & HDM-224 & H. lupulus & United States, Oregon & 2008 & JF304681 & JF304715 & JF414566 \\
\hline P. humuli h,e $^{\mathrm{e}}$ & HDM-247 & H. lupulus & United States, Washington & 2008 & JF304682 & JF304716 & JF414567 \\
\hline P. humuli ${ }^{\mathrm{d}}$ & HDM-254 & H. lupulus & United States, Oregon & 2008 & JF304683 & JF304717 & JF414568 \\
\hline P. humuli ${ }^{\mathrm{d}}$ & HDM-257 & H. lupulus & United States, Oregon & 2008 & JF304684 & JF304718 & JF414569 \\
\hline P. humuli & HDM-263 & H. lupulus & Czech Republic, Chrastany & Unknown & JF304685 & JF304719 & JF414570 \\
\hline P. humuli & HDM-266 & H. lupulus & Czech Republic, Kolesovice & Unknown & JF304686 & JF304720 & JF414571 \\
\hline P. humuli & SMK19582 & H. japonicus & Korea, Pyongchang & 2003 & JF314769 & JF304721 & JF414565 \\
\hline P. celtidis & SMK17780 & Celtis sinensis & Korea, Dongduchon & 2000 & JF314768 & $\ldots$ & JF414536 \\
\hline P. urticae ${ }^{\mathrm{f}}$ & HV713 & Urtica dioica & Austria, Oberösterreich & Unknown & $\ldots$ & DQ361163 & $\ldots$ \\
\hline Phytophthora infestans ${ }^{\mathrm{f}}$ & P106050 & Solanum tuberosum & Mexico & Unknown & $\ldots$ & EU079633.1 & $\ldots$ \\
\hline P. infestans ${ }^{\mathrm{f}}$ & INF-PO & S. tuberosum & Italy & Unknown & AJ854292 & $\ldots$ & $\ldots$ \\
\hline P. infestans $\mathrm{f}$ & Genome & S. tuberosum & Unknown & Unknown & $\ldots$ & $\ldots$ & U17009.2 \\
\hline
\end{tabular}

a ITS = internal transcribed spacer and $\operatorname{cox}=$ cytochrome $c$ oxidase.

${ }^{\mathrm{b}} P$. cubensis isolate used in host-specificity experiments on hop Nugget.

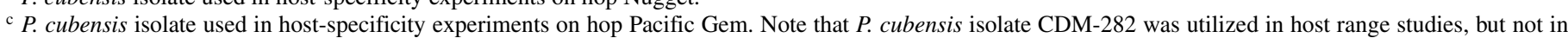
phylogenetic studies.

${ }^{d} P$. humuli isolate used in host-specificity experiments on cucumber Straight 8.

e $P$. humuli isolate used in host-specificity experiments on cantaloupe Ananes Yokneam.

${ }^{f}$ Sequences from National Center for Biotechnology Information GenBank.

g Complete mitochondrion genome. 
the first centrifugation. DNA was stored in TE buffer (S5 from MoBio or $10 \mathrm{mM}$ Tris and $1 \mathrm{mM}$ EDTA, $\mathrm{pH} 8.0$ ) at $-20^{\circ} \mathrm{C}$.

PCR amplification, cloning, and sequencing. Two nuclear loci and one mitochondrial locus were sequenced for phylogenetic analysis. The ITS of the nuclear ribosomal DNA (nrDNA) region, the nuclear $\beta$-tubulin gene $(\beta-t u b)$, and the mitochondrial cytochrome $c$ oxidase (cox) cluster (partitioned into $\operatorname{cox} 2$, $\operatorname{cox} 2$ coxl spacer, and $\operatorname{cox} l$ ) were amplified with the primers specified in Table 2. PCR reactions were carried out in a total volume of $25 \mu \mathrm{l}$ containing $12 \mu \mathrm{l}$ of PCR-grade water, $10 \mu \mathrm{l}$ of Hot Master Mix (5 PRIME, Gaithersburg, MD), $0.75 \mu \mathrm{l}(0.5 \mu \mathrm{M})$ of each forward and reverse primer, $0.5 \mu \mathrm{l}$ of acetonitrile $(50 \%$ by volume), and $1 \mu \mathrm{l}$ of template. The amplification program consisted of an initial denaturation at $94^{\circ} \mathrm{C}$ for $2 \mathrm{~min}$; followed by 40 cycles of denaturation at $94^{\circ} \mathrm{C}$ for $20 \mathrm{~s}$, annealing temperature specific for each primer pair (Table 2) for $30 \mathrm{~s}$, and extension at $65^{\circ} \mathrm{C}$ for $1 \mathrm{~min}$; with a final extension at $70^{\circ} \mathrm{C}$ for $10 \mathrm{~min}$. DNA fragments were electrophoresed in a $1 \%$ Tris-acetate EDTA gel. Ethidium bromide $\left(0.5 \mu \mathrm{g} \mathrm{m}^{-1}\right)$ was added to each gel, and the DNA fragments were visualized over a UV transilluminator.

Monosporangial isolates of $P$. humuli were directly sequenced in both directions from the PCR product. To obtain a single haplotype from non-monosporangial isolates, which could represent multiple individuals, the PCR products for each locus were cleaned as follows: amplicons were cleaned using the Amicon or Microcon centrifugal filters (Millipore), ligated into p-GEM TEasy vector (Promega Corporation, Madison, WI), and cloned in Escherichia coli strain DH5 $\alpha$ in accordance with the manufacturer's instructions. The insert and a portion of the vector were amplified using plasmid primers M13F and M13R. DNA fragments were electrophoresed and visualized as described above for confirmation before sequencing. Amplicons from PCR or clones were sequenced bidirectionally by the Center for Genome Research and Biocomputing on an ABI Prism 3730 Genetic Analyzer (Oregon State University, Corvallis). Typically, a single clone was chosen for each combination of host and locus to prevent the potential sequencing of multiple individuals in the non-monosporangial isolates. Most clones were sequenced one time (bidirectionally), unless there was ambiguity in the sequence, in which case sequencing was repeated until the sequence could be determined conclusively.

Sequence alignment and phylogenic analysis. Sequences were aligned in BioEdit (version 7.0.9.0; Ibis Therapeutics,
Carlsbad, CA) initially using ClustalW (61) under default settings followed by manual adjustments as needed. Complete sequences of Phytophthora infestans was available for all loci used in this study, and these sequences were downloaded from the National Center for Biotechnology Information (NCBI) GenBank to provide an outgroup to the Pseudoperonospora spp. for phylogenetic analyses (Table 1). Additionally, Pseudoperonospora urticae and $P$. celtidis sequence data were included as outgroups more closely related to $P$. cubensis and $P$. humuli for the nuclear (ITS and $\beta$-tub) and mitochondrial (cox cluster) analyses, respectively. The $P$. urticae sequences of ITS and $\beta$-tub were downloaded from the NCBI GenBank (Table 1).

MrModeltest 2.3 (38) was used to obtain an appropriate model of nucleotide substitutions. Models were chosen based on the Akaike information criterion. The best models for the nuclear and mitochondrial data sets were the Hasegawa, Kishino, and Yano model with gamma-distributed substitution rate $(\mathrm{HKY}+\mathrm{G})$ and the general time-reversible model with a proportion of invariant nucleotide sites $(\mathrm{GTR}+\mathrm{I})$, respectively. Incongruence length difference (ILD) tests were performed on the suitability of analyzing the cox cluster as a locus and for concatenating the nuclear loci for analysis using PAUP* version $4.0 \mathrm{~b} 10$ (58). Phylogenic analyses were performed using MrBayes version 3.1.2 $(25,47)$ for Bayesian analyses and RAxML version 7.2.5 (56,57) for maximum likelihood analysis. In the Bayesian analysis, three heated (temperature $=0.2$ ) and one cold simultaneous Markov chains were run for three million generations, saving a tree every 100 generations. Among these, the first 7,500 trees were ignored. A $50 \%$ majority rule consensus of the remaining trees was constructed by MrBayes version 3.1.2 to obtain estimates for the posterior probabilities of groups. To test the reproducibility of our results, the analyses were repeated three times, starting with random trees and default parameter values.

Phylogenetic analyses were conducted using RAxML version 7.2.5 under default parameters for the nucleotide substitution model with a rapid bootstrapping (1,000 replicates) via the CIPRES web portal. Additionally, the option to print branch lengths (-k) and to perform a rapid bootstrap analysis and search for the best-scoring maximum likelihood (ML) tree in one single program run (-f a) were selected. PAUP* version $4.0 \mathrm{~b} 10$ was used to create a $50 \%$ consensus tree from the files with the 1,000 bootstrap trees from RAxML version 7.2.5. For each locus and for the concatenated data set, the distance tree from the Bayesian

TABLE 2. Polymerase chain reaction and sequencing primers used in this study for different sequenced loci

\begin{tabular}{|c|c|c|c|c|c|}
\hline Primer & Sense & Sequence $\left(5^{\prime}-3^{\prime}\right)$ & Locus $^{\mathrm{a}}$ & Annealing temperature $\left({ }^{\circ} \mathrm{C}\right)$ & Source \\
\hline ITS1 & Forward & TCCGTAGGTGAACCTGCGG & ITS & 51 & 65 \\
\hline ITS4 & Reverse & GCATATCAATAAGCGGAGGA & ITS & 51 & 65 \\
\hline HDM07 & Forward & AGAATTGACTGCGAGTCC & ITS & 58.4 & 14 \\
\hline HDM04 & Reverse & AGCCACACAACACATAGT & ITS & 58.4 & 14 \\
\hline bTub136-OW & Forward & CGCATCAAYGTRTACTACAAYG & $\beta-T u b$ & 47 & 17 \\
\hline bTub1024R-OW & Reverse & CGAAGTACGAGTTCTTGTTC & $\beta-T u b$ & 47 & 17 \\
\hline bTubMM254-276R & Reverse & GTGATCTGGAAACCCTGCA & $\beta-T u b$ & 60 & This study \\
\hline bTubMM202-220F & Forward & ATTGACTCGGTGCTTGACG & $\beta-T u b$ & 60 & This study \\
\hline bTubMM558-576R & Reverse & GTGATACCAGACATGGCG & $\beta-T u b$ & 60 & This study \\
\hline bTubna492F & Forward & CATTTGCTTCCGCACACTTA & $\beta-T u b$ & 60 & This study \\
\hline FM35 & Forward & CAGAACCTTGGCAATTAGG & $\operatorname{cox} 2$ & 56 & 32 \\
\hline FM36 & Reverse & CAAATTTCACTACATTGTCC & $\operatorname{cox} 2$ & 56 & 32 \\
\hline FMPh-8b & Forward & AAAAGAGAAGGTGTTTTTTATGGA & $\operatorname{cox} 2$ - $\operatorname{cox} 1$ spacer & 56 & 34 \\
\hline FMPh-10b & Reverse & GCAAAAGCACTAAAAATTAAATATAA & $\operatorname{cox} 2-\operatorname{cox} 1$ spacer & 56 & 34 \\
\hline FM84 & Forward & TTTAATTTTTAGTGCTTTTGC & $\operatorname{cox} 1$ & 56 & 33 \\
\hline FM85 & Reverse & AACTTGACTAATAATACCAAA & $\operatorname{cox} 1$ & 56 & 33 \\
\hline FM85RC ${ }^{b}$ & Reverse & TTTGGTATTATTAGTCAAGTT & $\operatorname{cox} 1$ & 56 & 33 \\
\hline FM77 & Reverse & CACCAATAAAGAATAACCAAAAATG & $\operatorname{cox} 1$ & 56 & 33 \\
\hline FM83 & Reverse & 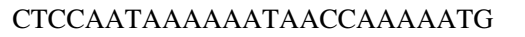 & $\operatorname{cox} 1$ & 56 & 33 \\
\hline M13F & Forward & TGTAAAACGACGGCCAGT & Plasmid & 50 & $\ldots$ \\
\hline M13R & Reverse & CAGGAAACAGCTATGACC & Plasmid & 50 & $\ldots$ \\
\hline
\end{tabular}

${ }^{a}$ ITS $=$ internal transcribed spacer, $\beta$-Tub $=\beta$-tubulin, and $c o x=$ cytochrome $c$ oxidase.

b FM85RC is the reverse compliment of FM85 (33). 
analysis was used as the template to reconcile the trees from each analysis to form a consensus tree with $50 \%$ majority rule for the trees inferred by both programs.

An additional phylogenetic analysis was performed incorporating ITS sequences obtained in this study with all available published $P$. cubensis and $P$. humuli sequences in the NCBI database to provide a larger data set, including isolates from Europe and Asia (Table 3). P. celtidis, P. urticae, and P. cannabina ITS sequences were included as additional outgroups. Three sequences from China were 709 bp long; therefore, the other sequences were shortened accordingly for the phylogenetic analysis. Phylogeny was inferred by RAxML version 7.2.5 (same conditions as above) and MrBayes version 3.1.2 using the general time-reversible nucleotide model with gamma distributed substitution rate $(\mathrm{GTR}+\mathrm{G})$ found by MrModeltest version 2.3. The $50 \%$ consensus trees were reconciled using the tree inferred by Bayesian analysis as the template. Trees were presented using TreeView version 1.6.6 (39) with Phytophthora infestans as the outgroup.

Host range study. Pseudoperonospora humuli isolates from Oregon (three isolates) and Washington (one isolate) and $P$. cubensis isolates from North Carolina (five isolates), Michigan (two isolates), Ohio (one isolate), and South Carolina (one isolate) were used for a host range study conducted during 2008 to 2010 (Table 1). The pathotype of three of the P. cubensis isolates was determined previously (10), each being considered a unique pathotype. During a given inoculation experiment, $P$. humuli was spray inoculated as described above onto three cucumber plants (Straight 8) at a concentration of $5 \times 10^{3}$ spores $\mathrm{ml}^{-1}$ while $P$. cubensis was spray inoculated onto three hop plants (Nugget) at a concentration of $5 \times 10^{3}$ spores $\mathrm{ml}^{-1}$. Cucumber and hop plants were trimmed to three nodes per plant (nodes 3 to 7 , depending on plant age and leaf quality). A positive control and negative (water-only) control plant was included for each Pseudoperonospora spp. in every experiment. All the plants were grown in $440-\mathrm{cm}^{3}$ pots and put into Pasta Keeper containers. The plants were incubated as described above for $P$. cubensis on cucumber. All but three of the pathogen strains were evaluated at least twice for both host combinations.

Additionally, host range studies were conducted on Pacific Gem hop and Ananes Yokneam melon (cantaloupe), which are regarded as universally susceptible to $P$. humuli and $P$. cubensis, respectively (29). In the Pacific northwestern United States, Pacific Gem plants are killed by downy mildew because of their

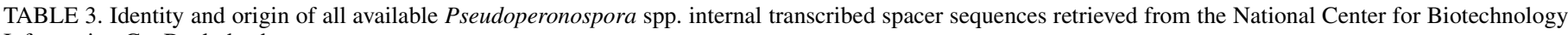
Information GenBank database

\begin{tabular}{|c|c|c|c|c|}
\hline Organism & Isolate & Host & Origin & GenBank accession no. \\
\hline Pseudoperonospora cannabina & MZM71018 & Cannabis sativa & Latvia & AY608612 \\
\hline$P$. celtidis & SMK17780 & Celtis sinensis & Korea & AY608613 \\
\hline P. cubensis & HV222 & Cucumis sativus & Austria & AY198306 \\
\hline P. cubensis & HV2279 & Impatiens irvingii & Cameroon & EU660054 \\
\hline P. cubensis & JinShan & C. sativus & China & DQ025515 \\
\hline P. cubensis & MinHang & C. sativus & China & DQ025516 \\
\hline P. cubensis & PuDong & C. sativus & China & DQ025517 \\
\hline P. cubensis & Not available & C. sativus & China & AY744946 \\
\hline P. cubensis & JM_12/00 & C. sativus & Czech Republic & EU876600 \\
\hline P. cubensis & JM_39/01 & Unknown & Czech Republic & EU876601 \\
\hline P. cubensis & Leb_4/95 & C. sativus & Czech Republic & EU876604 \\
\hline P. cubensis & OL_1/88 & C. sativus & Czech Republic & EU876599 \\
\hline P. cubensis & OL_26/01a & Unknown & Czech Republic & EU876598 \\
\hline P. cubensis & SC_75/01 & Unknown & Czech Republic & EU876603 \\
\hline P. cubensis & ZL_35/01 & Unknown & Czech Republic & EU876602 \\
\hline P. cubensis & WE_3/00 & C. sativus & France & EU876597 \\
\hline P. cubensis & CEC__2811 & C. sativus & Greece & EU876592 \\
\hline P. cubensis & CEC_2812 & C. sativus & Greece & EU876591 \\
\hline P. cubensis & CEC_2813 & C. sativus & Greece & EU876590 \\
\hline P. cubensis & CEC_2814 & C. sativus & Greece & EU876589 \\
\hline P. cubensis & CEC_2815 & C. sativus & Greece & EU876588 \\
\hline P. cubensis & CEC_2816 & C. sativus & Greece & EU876587 \\
\hline P. cubensis & CEC_2818 & C. sativus & Greece & EU876586 \\
\hline P. cubensis & CEC_2819 & C. sativus & Greece & EU876585 \\
\hline P. cubensis & CEC_2820 & C. sativus & Greece & EU876584 \\
\hline P. cubensis & HIE_2409 & C. sativus & Greece & EU876596 \\
\hline P. cubensis & HIE_2410 & C. sativus & Greece & EU876595 \\
\hline P. cubensis & HIE_2412 & C. sativus & Greece & EU876594 \\
\hline P. cubensis & HIE_2413 & C. sativus & Greece & EU876593 \\
\hline P. cubensis & SMK 11284 & C. melo & Korea & AY608614 \\
\hline P. cubensis & SMK12174 & C. sativus & Korea & AY608616 \\
\hline P. cubensis & SMK13288 & Cucurbita moschata & Korea & AY608619 \\
\hline P. cubensis & SMK14235 & Citrullus vulgaris & Korea & AY608618 \\
\hline P. cubensis & SMK15170 & Cucumis melo & Korea & AY608615 \\
\hline P. cubensis & SMK18951 & C. sativus & Korea & AY608617 \\
\hline P. cubensis & SMK19205 & Cucurbita moschata & Korea & AY608620 \\
\hline P. cubensis & SMK21327 & Lagenaria siceraria & Korea & DQ409815 \\
\hline P. cubensis & D2 & Trichosanthes cucumerina & Malaysia & GU233293 \\
\hline P. cubensis & Not available & Unknown & Taiwan & EF050035 \\
\hline P. humuli & HV136 & Humulus lupulus & Austria & AY198304 \\
\hline P. humuli & HV148 & H. lupulus & Austria & AY198305 \\
\hline P. humuli & Not available & H. lupulus & Czech Republic & AF448225 \\
\hline P. humuli & SMK11608 & H. japonicus & Korea & AY608621 \\
\hline P. humuli & SMK11675 & H. lupulus & Korea & AY608624 \\
\hline P. humuli & SMK18856 & H. japonicus & Korea & AY608622 \\
\hline P. humuli & SMK19582 & H. japonicus & Korea & AY608623 \\
\hline P. urticae & HV715 & Urtica dioica & Korea & AY 198307 \\
\hline
\end{tabular}


extreme susceptibility to the disease. The cucurbit species was changed to Ananes Yokneam cantaloupe to verify that the host specificity of $P$. humuli observed on cucumber (described below) was consistent on another cucurbit host.

The experiments were rated 7 and 14 days postinoculation by stereomicroscopic examination of the abaxial surface of each leaf. Each leaf was rated for a hypersensitive response (HR) (localized water soaking, chlorosis, and necrosis) and sporulation. The rating on the 7 th day was done without harming the plant, whereas the day-14 rating was destructive. For 15 of 18 isolatehost combinations in which sporulation was observed, sporangiophores and sporangia were counted or were estimated if individual spore structures were too numerous to identify.

To confirm that the infection was produced by the organism inoculated (i.e., P. cubensis on hop leaves), sporangia were collected from studies on two strains ( $P$. cubensis isolates CDM-255 and CDM-276 on hop) and identified using the cox2 locus. Cox2 was chosen due to the relative ease in amplification and the presence of four well-conserved single-nucleotide polymorphisms
(SNPs) that differentiate $P$. humuli and $P$. cubensis among the isolates used in this study with only three exceptions. The exceptions are isolates SMK19582 (P. humuli from H. japonicus in Korea with three SNPs, only two of which are found in most $P$. cubensis); CDM-241 (P. cubensis from squash in North Carolina, with one SNP found in most $P$. humuli); and CDM-248 (P. cubensis from acorn squash in North Carolina, with three SNPs found in most $P$. humuli). None of these isolates were alive when the host-specificity experiments were performed, nor would it be plausible that the DNA from the three aberrant isolates would contaminate the DNA from the host-specificity experiments because the host range studies and DNA extractions were not conducted at the same time.

To further confirm that the infection was produced by the organism inoculated onto hop and cucumber, sporangia of $P$. cubensis produced on hop were tested on hop and cucumber to confirm that the pathogen had a similar pathogenicity pattern as $P$. cubensis when inoculated on cucumber. For this experiment, $P$. cubensis (CDM-255) from Straight 8 cucumber was spray inocu-

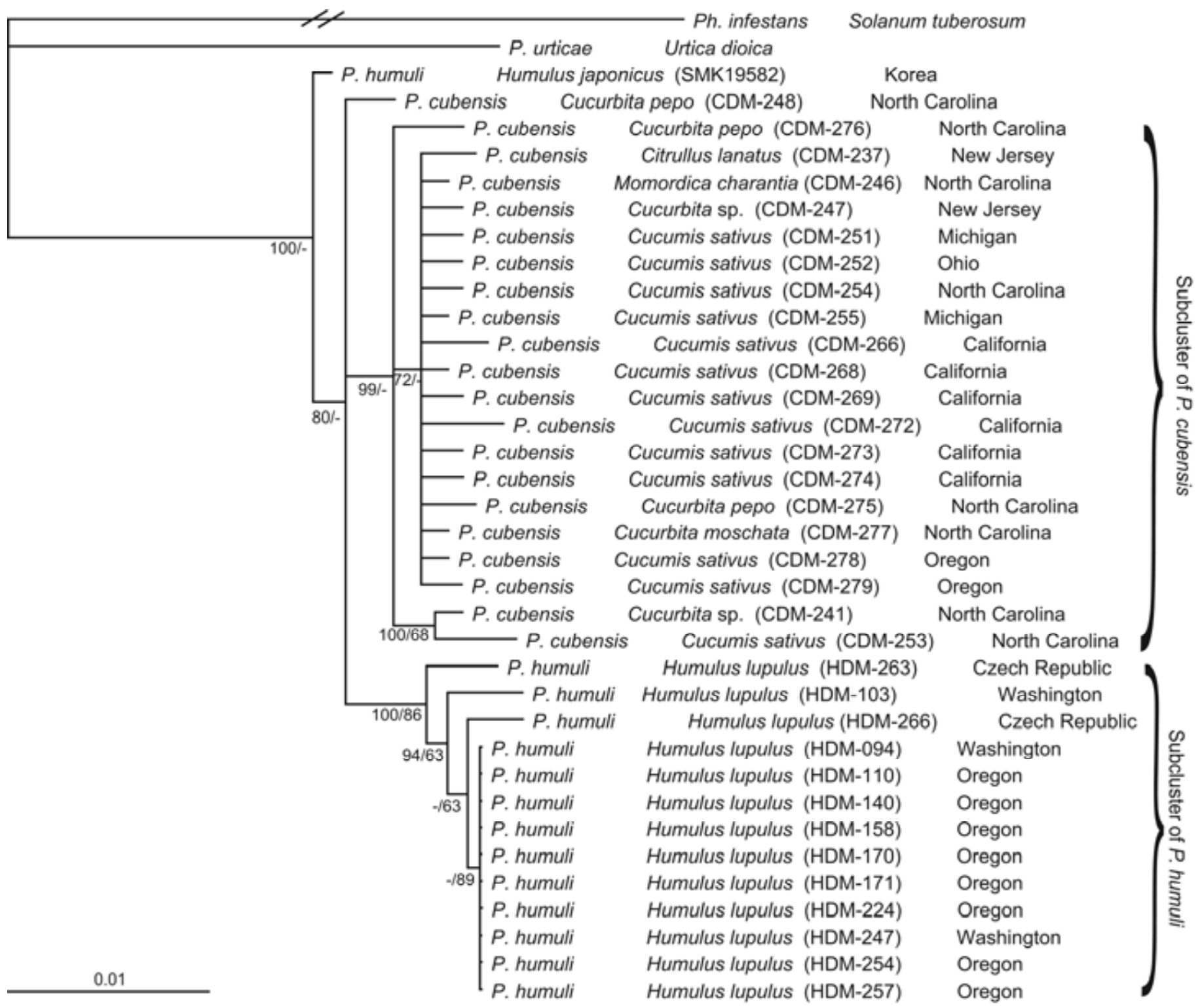

Fig. 1. A $50 \%$ consensus tree of Bayesian and maximum likelihood analyses of an alignment of the internal transcribed spacer and $\beta$-tubulin loci for Pseudoperonospora cubensis and P. humuli. Genetic distances were computed according to the Hasegawa, Kishino, and Yano model, additionally assuming a gamma-distributed substitution rate. Tree topology was rooted with Phytophthora infestans. Numbers on branches are posterior probabilities $\left(3 \times 10^{6}\right.$ generations) followed by bootstrap support values (1,000 replicates). Dashes indicate support $<50 \%$. The expected number of nucleotide substitutions between taxa is represented by branch length and the scale bar equals the expected number of nucleotide substitutions per site. The interrupted root branch was scaled to onequarter its length. 
lated onto four hop plants (Pacific Gem) plants in $440-\mathrm{cm}^{3}$ pots at $5 \times 10^{5}$ sporangia $\mathrm{ml}^{-1}$ in the manner described above. The four plants were placed into a prepared barrel container and were incubated in the manner of $P$. cubensis. On the seventh day, the inoculum from the hop plants was collected, combined, and quantified. From this inoculum, four hop plants (Pacific Gem) and two cucumber plants (Straight 8 ) were spray inoculated at $5 \times 10^{3}$ sporangia $\mathrm{ml}^{-1}$. The plants were treated in the same way as the first four hop plants. On the seventh day, the inocula from the hop and cucumber plants were collected and quantified, and DNA was extracted for confirmation that the pathogen infecting these plants was P. cubensis.

\section{RESULTS}

Phylogenic analysis. We obtained sequence data for $21 \mathrm{P}$. $\mathrm{Cu}$ bensis, 14 P. humuli, 1 P. celtidis (cox cluster) or P. urticae (ITS and $\beta$-tub), and 1 (per analysis) Phytophthora infestans isolates. Overall, the phylogenetic analyses included 809 bp of the ITS, consisting of the complete ITS region (ITS1, 5.8S nrDNA, and ITS2), $696 \mathrm{bp}$ of $\beta$-tub, and 2,067 bp of the cox cluster (673 bp of partial cox2, 204 bp of cox2-cox 1 spacer, and 1,190 bp of partial coxl). The nuclear data set, consisting of the ITS and $\beta-t u b$, contained $1,555 \mathrm{bp}$, of which there were 41 parsimony-informative characteristics, 205 that were parsimony uninformative, and 1,309 that were constant. For the cox cluster, there were 56 parsimony-informative sites, 284 that were parsimony uninformative, and 1,727 that were constant. For some isolates, the entire ITS, $\beta$-tub, and coxl loci did not amplify; therefore, alternate primers were used (ITS or coxl) or designed $(\beta-t u b)$ to amplify overlapping smaller fragments, which were then aligned to obtain a sequence for the whole region (Table 2). The $\operatorname{cox} 2$ gene and the cox2-coxl spacer amplified well for all isolates used in this study.

The ILD test showed no significant difference between the loci within the cox cluster $(P=0.774)$ or between ITS and $\beta$-tub $(P=$ $0.951)$, indicating that the loci within these data sets were congruent and could be analyzed with single-nucleotide substitution models. Additionally, the relatively conserved topology

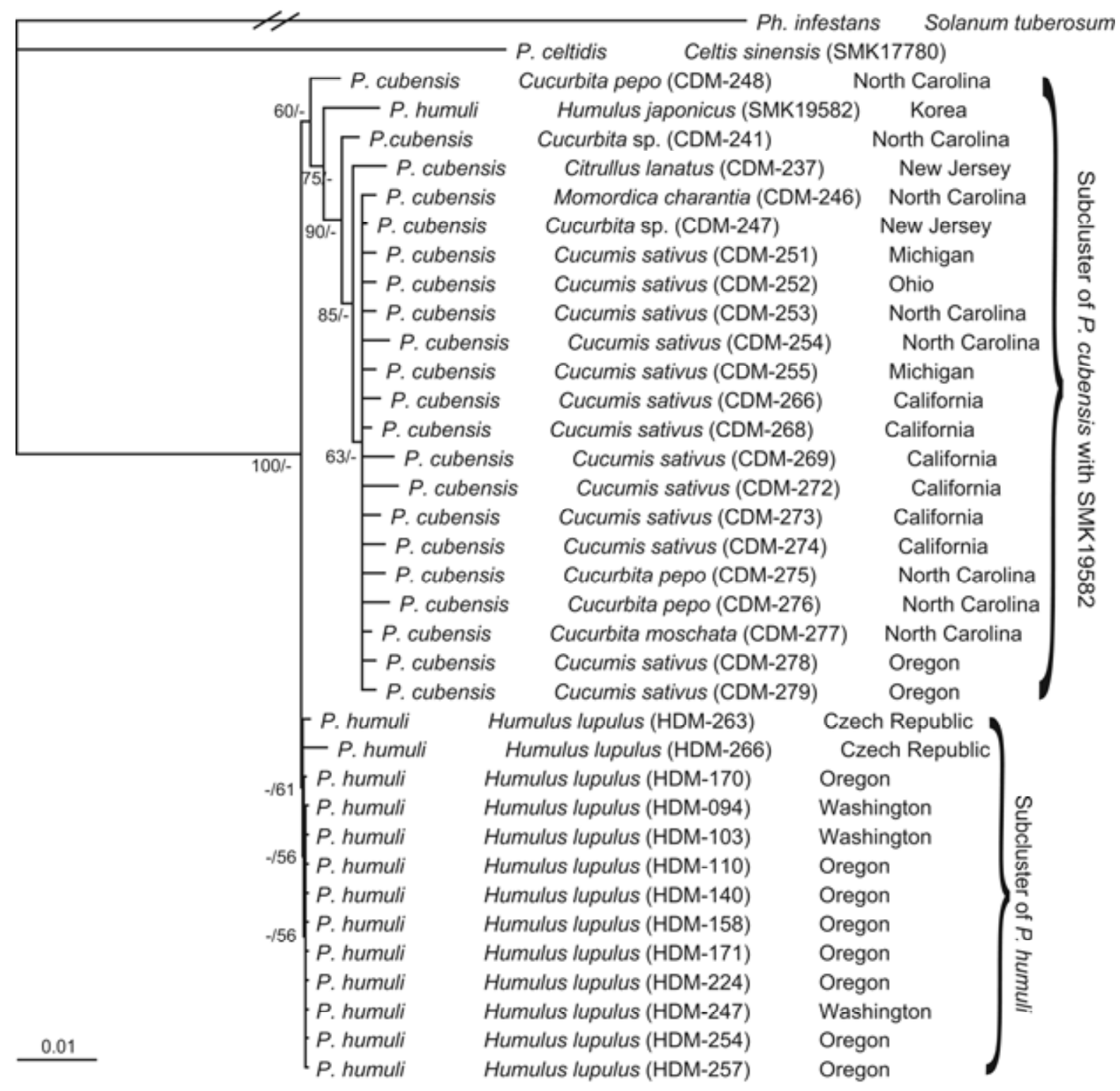

Fig. 2. A 50\% consensus tree of Bayesian and maximum likelihood analyses of an alignment of the cytochrome $c$ oxidase (cox) cluster region (partial cox2, cox2coxl spacer, and partial coxl) for Pseudoperonospora cubensis and P. humuli. Genetic distances were computed according to the general time-reversible model, additionally assuming a proportion of invariant nucleotide sites. The tree was rooted with Phytophthora infestans. Numbers on branches are posterior probabilities $\left(3 \times 10^{6}\right.$ generations) followed by bootstrap support values $(1,000$ replicates). Dashes indicate support $<50 \%$. The expected number of nucleotide substitutions between taxa is represented by branch length and the scale bar equals the expected number of nucleotide substitutions per site. The interrupted root branch length was scaled to one-half its length. 
(and statistical support) of the trees inferred by the individual loci suggested that concatenation of the loci into nuclear and mitochondrial data sets was appropriate. The phylogenetic relationships between Pseudoperonospora cubensis and $P$. humuli inferred from Bayesian analysis and heuristic ML analysis (performed by RAxML) of the aligned nucleotide sequences are shown for the nuclear loci (Fig. 1), cox cluster (Fig. 2), and ITS nrDNA sequence data downloaded from GenBank (Fig. 3). All four Bayesian analyses resulted in the same tree topology with almost identical posterior probability (PP) values for the analysis of the cox cluster and nuclear loci. The topology differed slightly for two of the four Bayesian analyses of the ITS nrDNA sequence data downloaded from GenBank in that the cluster of $P$. cubensis isolates from this study, Europe, and some from Asia was not resolved. Also, the GenBank sequence for one isolate of $P$. humuli on $H$. japonicus (SMK19582) clustered basally to the $P$. humuli$P$. cubensis isolates. The PP values were nearly identical for the unchanged clusters.

The trees inferred by nuclear loci agreed on the separation of most of the $P$. humuli isolates from the $P$. cubensis isolates. A cluster of all the $P$. humuli isolates, with the exception of the $H$. japonicus from Korea (SMK19582), was well supported by both

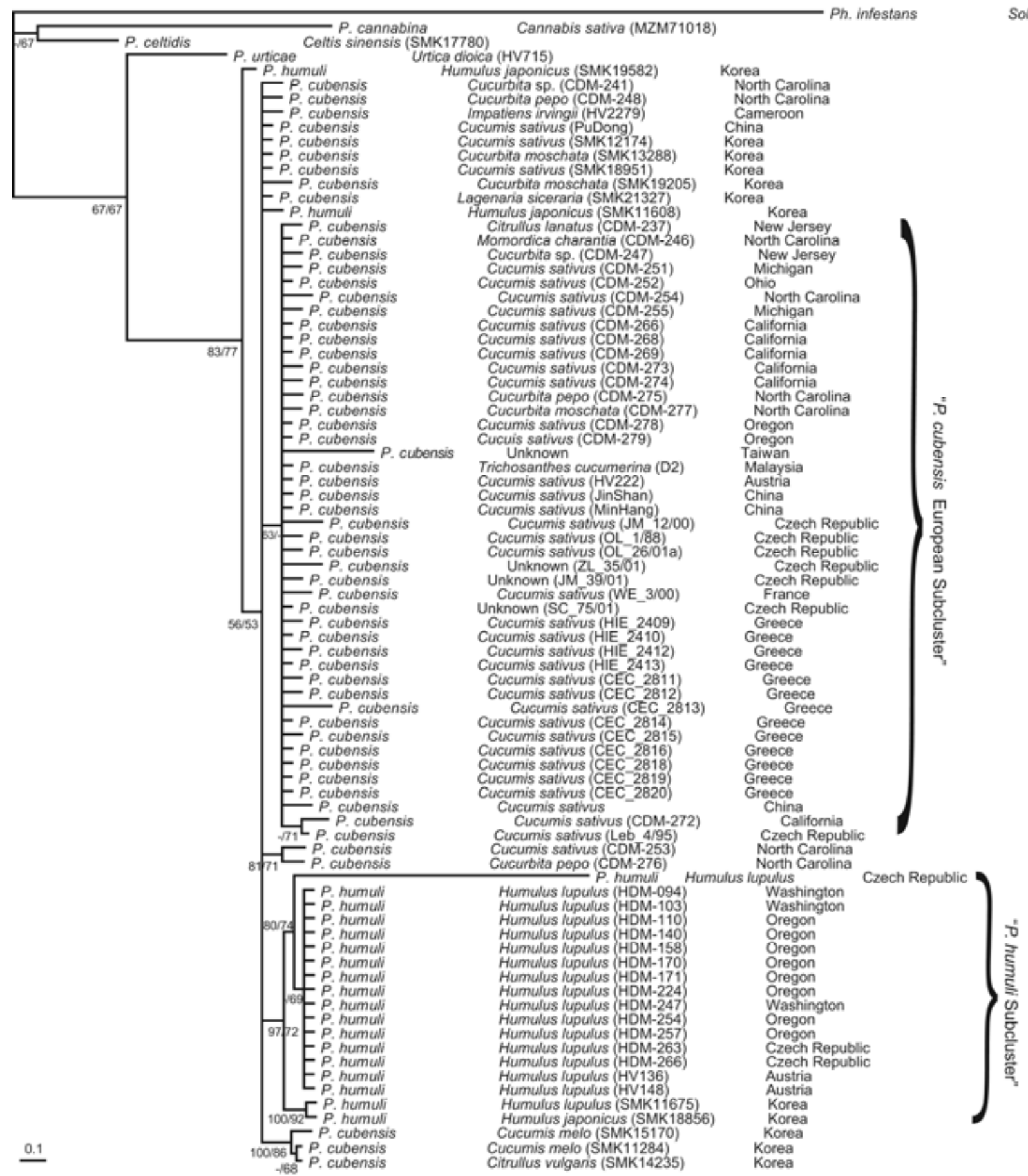

Fig. 3. A 50\% consensus tree of Bayesian and maximum likelihood analyses of an alignment of published Pseudoperonospora spp. internal transcribed spacer sequences from GenBank. Genetic distances were computed according to the general time-reversible model, additionally assuming a gamma-distributed substitution rate. The tree was rooted with Phytophthora infestans. Numbers on branches are posterior probabilities $\left(3 \times 10^{6}\right.$ generations) followed by bootstrap support values $(1,000$ replicates). Dashes indicate support $<50 \%$. The expected number of nucleotide substitutions between taxa is represented by branch length and the scale bar equals the expected number of nucleotide substitutions per site. 
the Bayesian and ML analyses (Fig. 1; 100\% PP, 86\% bootstrap support [BS]). Only the Bayesian analysis resolved the relationships within the $P$. cubensis isolates, with a cluster of all $P$. cubensis isolates except one from Cucurbita pepo from North Carolina (CDM-248) supported with 99\% PP.

The consensus trees of the cox cluster inferred by Bayesian and ML analyses separated the $P$. cubensis isolates from the $P$. humuli isolates, with the exception of the Korean $P$. humuli isolate (Fig. 2 ). The separation of either cluster from the other group was supported in the Bayesian analysis $100 \%$ but not in terms of BS. A cluster of $P$. cubensis isolates exclusive of an isolate on $C$. pepo from North Carolina (CDM-248) and the Korean P. humuli isolate had $90 \%$ PP.

The trees inferred by ML and Bayesian analysis of the ITS region that included all published Pseudoperonospora sequences in GenBank had similar topology (Fig. 3). Both analyses supported a clade of $P$. cubensis and $P$. humuli subtended by the other Pseudoperonospora spp. (83\% PP, $77 \%$ BS). A cluster of all the $P$. humuli isolates except for two isolates from $H$. japonicus from Korea (SMK11608 and SMK19582) was found with both analyses (97\% PP, 72\% BS). Two subclusters were defined within the P. humuli cluster (80 and $100 \%$ PP, 74 and $92 \%$ BS) separating U.S. and European isolates from those originating from Korea. Within the $P$. cubensis clade, a cluster of three isolates from Cucumis melo and Citrullus vulgaris from Korea was supported by both analyses (100\% PP, $86 \%$ BS), including a cluster of two isolates sampled in this study (CDM-253 and CDM-276; $81 \%$ PP, $71 \%$ BS).

Host range study. $P$. cubensis isolates inoculated on hop plants sporulated on 48 of 61 replicate plants (79\%) (Table 4). Isolates of $P$. humuli inoculated onto cucurbit plants sporulated on only 1 of 33 replicate plants (3\%) (Table 4), and the sporulation consisted of a single sporangiophore. The positive control plants for both pathogens were always characterized by profuse sporulation but never localized necrosis. No sporulation or localized necrosis was observed in any of the negative control plants. In contrast, the inoculated leaves developed scattered areas of localized necrosis when inoculated with the reciprocal pathogen (Figs. 4 and 5). We consider the localized necrosis to be HR-like if the necrosis was localized to a few cells rather than dispersed and spreading, as typified in the positive controls. When sporulation was observed, the sporangiophores invariably emerged from the center or the inside edge of a chlorotic or, more commonly, necrotic lesion (Fig. 5). Typically, fewer than half of the HR-like lesions contained sporangiophores when hop plants were inoculated with $P$. cubensis. The proportion of lesions with sporangiophores appeared to be greater with Pacific Gem than with Nugget, although no attempt was made to quantify this observation (Table 4).

Differences in virulence were seen both with $P$. cubensis on the two hop cultivars and with $P$. humuli on the two universally susceptible species of cucurbits. $P$. cubensis sporulated relatively more profusely on Pacific Gem than on Nugget (Table 4), and more HR-like lesions were seen on Pacific Gem. However, this observation is limited because only one isolate of $P$. cubensis (CDM-252) was inoculated onto both of the hop cultivars. For the cucurbit species, Ananes Yokneam cantaloupe had more necrosis whether infected with $P$. cubensis or $P$. humuli compared with Straight 8 cucumber. When inoculated with $P$. humuli, cantaloupe had more HR-like lesions than cucumber. The one cucurbit plant on which $P$. humuli sporulated was a cantaloupe in which there was one sporangiophore bearing four sporangia emerging from near the center of a necrotic HR-like lesion on the 7-day rating.

PCR analysis of the inoculum harvested from hop infected with $P$. cubensis verified that the pathogen contained SNPs in the cox2 region which correspond with all but two $P$. cubensis isolates (CDM-241 and CDM-248; see above). In reinoculation experiments to further verify that the sporulation on hop plants was indeed $P$. cubensis, the first set of four Pacific Gem hop plants yielded $\approx 7.4 \times 10^{4}$ sporangia/plant. The same isolate inoculated onto two Straight 8 cucumber plants yielded $>4.17 \times 10^{6}$ sporangia/plant. The hop plants displayed symptoms similar to those seen in the host-specificity experiments but had more HR lesions, presumably due to the higher concentration of sporangia used for inoculation. The second set of Pacific Gem plants had symptoms similar to those seen in the host-specificity experiments (Fig. 6) and yielded $\approx 5 \times 10^{4}$ sporangia/plant. The two cucumber plants (Straight 8) inoculated with inoculum from the first set of hop plants yielded $\approx 4.5 \times 10^{6}$ sporangia/plant. The cucumber exhibited typical disease symptoms for cucumber plants inoculated with P. cubensis (Fig. 6A and C). However, the sporulation, while abundant, was not as profuse as in many of the positive-control cucumber plants in the host-specificity experiments, perhaps due to the inoculum coming from a poor host.

\section{DISCUSSION}

For each of the sequence data sets, the resulting trees consistently divided isolates of $P$. cubensis from isolates of $P$. humuli into separate clades, with the exception of $P$. humuli on $H$. japonicus from Korea (SMK19582 and SMK11608). The nuclear loci and ITS sequence analyses strongly supported a cluster of the majority of $P$. humuli isolates with both the Bayesian and ML analyses. Within the $P$. cubensis clusters, there did not appear to be a significant genetic difference between isolates from the eastern United States and those from the western United States.

Other work that focused on multiple isolates of $P$. cubensis and $P$. humuli primarily reconstructed phylogenies based on ITS sequence. Choi et al. (6) examined the phylogenetic relationship within the genus Pseudoperonospora by using ITS sequence data from nine isolates of each pathogen with Bayesian and maximum parsimony (MP) analyses. Sarris et al. (53) inferred a phylogeny of $P$. cubensis and $P$. humuli from ITS sequences using the neighbor-joining method with the isolates of Choi et al. (6) as well as 22 isolates of $P$. cubensis from the Czech Republic and

TABLE 4. Results of host-specificity experiments with Pseudoperonospora cubensis on two hop cultivars and $P$. humuli on two cucurbit species

\begin{tabular}{llcc}
\hline Isolate & Cultivar, species & $\begin{array}{c}\text { Proportions of } \\
\text { infections }^{\mathrm{a}}\end{array}$ & Sporangiophores/plant ${ }^{\mathrm{b}}$ \\
\hline P. cubensis & Nugget & $3 / 6$ & $38.6 \pm 28.5$ \\
CDM 251 & Nugget & $9 / 15$ & $36.5 \pm 33.4$ \\
CDM 252 & Nugget & $4 / 6$ & $0.3 \pm 0.5$ \\
CDM 253 & Nugget & $4 / 6$ & NF \\
CDM 255 & Pacific Gem & $6 / 6$ & $>600$ \\
CDM 252 & Pacific Gem & $3 / 3$ & $>900$ \\
CDM 254 & Pacific Gem & $6 / 6$ & $451.7 \pm 380$ \\
CDM 275 & Pacific Gem & $6 / 6$ & $239.2 \pm 183.0$ \\
CDM 276 & Pacific Gem & $2 / 2$ & $107.0 \pm 70.7$ \\
CDM 277 & Pacific Gem & $5 / 5$ & 0 \\
CDM 282 & Cucumber & $0 / 6$ & 0 \\
$P$. humuli & Cucumber & $0 / 6$ & 0 \\
HDM 224 & Cucumber & $0 / 6$ & 0 \\
HDM 247 & Cucumber & $0 / 6$ & 0 \\
HDM 254 & Cantaloupe & $0 / 3$ & 1 \\
HDM 257 & Cantaloupe & $1 / 6$ & \\
HDM 224 & CDM 247 & C & \\
\hline
\end{tabular}

a Proportion of successful infections was defined as the number of plant replicates with sporulation out of the total number of plant replicates for that isolate. Positive controls were characterized by profuse sporulation and negative controls were free of sporulation in every run of the experiments. The cucumber cultivar was Straight 8 and the cantaloupe cultivar was Ananes Yokneam.

b Number of sporangiophores per plant ( \pm standard error) was calculated only for those replicates for which the number of sporangiophores was enumerable at 14 days postinfection (dpi). NF = sporangiophores were not found at $14 \mathrm{dpi}$ but were found at $7 \mathrm{dpi}$. 
Greece and $1 P$. humuli isolate from the Czech Republic. Göker et al. (16) inferred the phylogenetic relationships of the same data set as Sarris et al. (53) with two additional P. cubensis isolates from Asia using RAxML and MP. In general, the resolution of the tree increased with each study and the topology of the trees remain fairly stable, although the statistical support for branches varied depending on the number of isolates and the type of analysis used $(6,16,49,53)$. The isolates of $P$. cubensis tend to be in a clade divided into three groups. Two of the groups are a subcluster with isolates of $P$. cubensis from Europe and China and a subcluster consisting of isolates from Korea $(6,16,49,53)$. The third group is composed of four $P$. cubensis isolates from Korea and two $P$. humuli isolates on $H$. japonicus from Korea of Choi et al. (6) that are not resolved into a clade but, instead, appear to be basal or adjacent to the two subclades $(6,16,53)$. The $P$. humuli isolates are partitioned into a separate cluster from $P$. cubensis (except the two $P$. humuli isolates from $H$. japonicus clustering with $P$. cubensis) composed of two subclades. The subclades divide the remaining $P$. humuli isolates from Korea clustering together from $P$. humuli isolates from Europe.

The tree based on ITS sequence data was similar in topology and support values to those of Göker et al. (16). All 14 P. humuli isolates from the western United States clustered with those from Europe, separate from a subcluster of $P$. humuli from Korea. The " $P$. humuli cluster" was well supported in these analyses, similar to Runge et al. (49) who used a different set of isolates from Europe and Argentina. All but four of the isolates of $P$. cubensis from the current study clustered with the "European subcluster" of Sarris et al. (53). However, this subclade was only supported in the Bayesian analysis with 63\% PP and was not found in two of the four Bayesian analyses. In the analyses of this study, isolates of $P$. cubensis on Cucumis sativus from China (PuDong), on $C$. sativus and Cucurbita spp. from North Carolina (CDM-241, 248, 253, and 276), and on Impatiens irvingii from Cameroon (HV2279) were not included in the "European subcluster."

Our analyses indicate differences both between and within $P$. cubensis and $P$. humuli. There appears to be a genetic divergence between the isolates from Korea and isolates from many other regions of the world in both the $P$. cubensis and $P$. humuli clusters (49). Two of the four $P$. humuli isolates from $H$. japonicus (SMK11608 and SMK19582) cluster with Korean P. cubensis isolates. Within the P. humuli cluster, the Korean isolates are strongly supported as being separate from both the European and American isolates. These differences could be explained by several factors. One obvious explanation is that the Pseudoperonospora sp. on $H$. japonicus is distinct from $P$. humuli. Isolates SMK11608 and SMK19582 are more $P$. cubensis-like and either cluster basally (49) or with $P$. cubensis. Another explanation could be that these observations are sampling artifacts based on two very different isolates. The differences may be due to increased genetic diversity in isolates collected in Korea compared with isolates collected in other locations around the world. Further sampling in Korea and other parts of Asia and molecular markers with greater resolution are needed to clarify

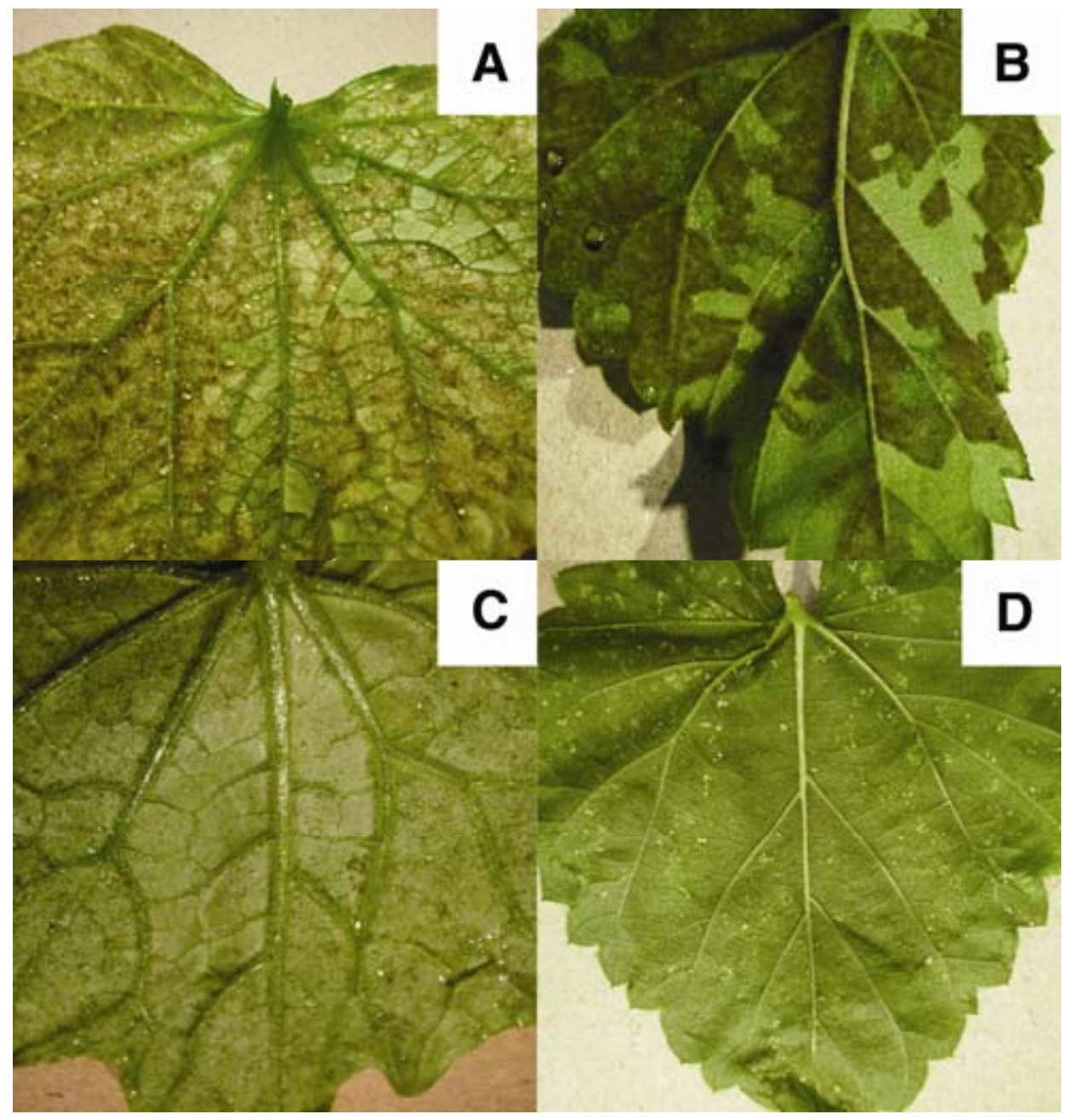

Fig. 4. Macroscopic signs and symptoms from host-specificity experiments with Pseudoperonospora cubensis and P. humuli. A, P. cubensis inoculated on cucumber 'Straight 8'; B, P. humuli inoculated on hop 'Pacific Gem'; C, P. humuli inoculated on Straight 8; and D, P. cubensis inoculated on Pacific Gem. A and $\mathbf{B}$, Positive controls are characterized by profuse sporulation and few hypersensitive-like lesions, whereas $\mathbf{C}$ and $\mathbf{D}$, the opposite is true of the reciprocal inoculations. 
these observations and the diversity within and among $P$. humuli on Humulus spp.

Hop downy mildew was first recorded in Japan in 1905 and it is possible that $P$. humuli originated in Asia (36). It is interesting to note that Asia, especially the areas of India, Indo-Malaysia, and China, is the presumed center of origin for the genus Humulus (37) as well as many genera and species of cucurbits, including $C$. sativus and several other Cucumis spp. (26,46). Citrullus spp., most Cucumis spp. (including C. melo), and Cucurbita spp. arose in Africa (Citrullus and Cucumis spp.) and South America (Cucurbita spp.) and some, but not all, of the P. cubensis isolates that were from these hosts (i.e., CDM-241, 248, and 276 as well as SMK11284, 14235, and 15170) did not cluster with the other $P$. cubensis isolates in the phylogenetic analyses, indicating separate origins.

Host-specificity experiments indicate that $P$. cubensis and $P$. humuli are biologically distinct, because $P$. cubensis appears to be able to infect the primary host of $P$. humuli, albeit at very low levels, whereas $P$. humuli was essentially unable to successfully infect two highly susceptible hosts of $P$. cubensis. This indicates that occasional host jumps appear possible, and suggests that a host jump may have occurred recently. Hop plants inoculated with $P$. cubensis sporulated in $79 \%$ of the replicates whereas $P$. humuli only sporulated once on a cucurbit host out of 33 replicate plants and, in that instance, only a single sporangiophore was observed.
Each host exhibited lesions consistent with an HR when inoculated with the reciprocal pathogen, and sporulation was observed in the center or inner edge of the necrotic, or occasionally chlorotic, lesion. It appears that at least two of the reportedly universal hosts of $P$. cubensis are extremely poor or, more likely, non-hosts of $P$. humuli under natural conditions; while the two hop cultivars tested, both highly susceptible to hop downy mildew, were also poor or non-hosts for P. cubensis. The existence of hop production in countries where cucurbit downy mildew occurs but not hop downy mildew (e.g., Australia, South Africa, and New Zealand) also circumstantially supports the idea that, under natural conditions, hop does not host $P$. cubensis at detectable levels. Hoerner (24) unsuccessfully attempted to infect unspecified ("all available") hosts of $P$. cubensis with $P$. humuli. Unfortunately, the experiments were undocumented aside from a passing remark (24); therefore, the conditions and exact organisms are unknown. Host jumps and close species boundaries have been observed for other oomycete pathogens; for example, in clade 1c, Phytophthora spp., including Phytophthora ipomoeae, $P$. mirabilis, and $P$. infestans, and are likely to be frequent in the downy mildews, as exemplified by the Hyaloperonospora genus $(21,59)$.

The ability of Pseudoperonospora cubensis to successfully infect and sporulate, albeit at low levels, on the two hop cultivars tested may be due to its polyphagic lifestyle. P. cubensis has been

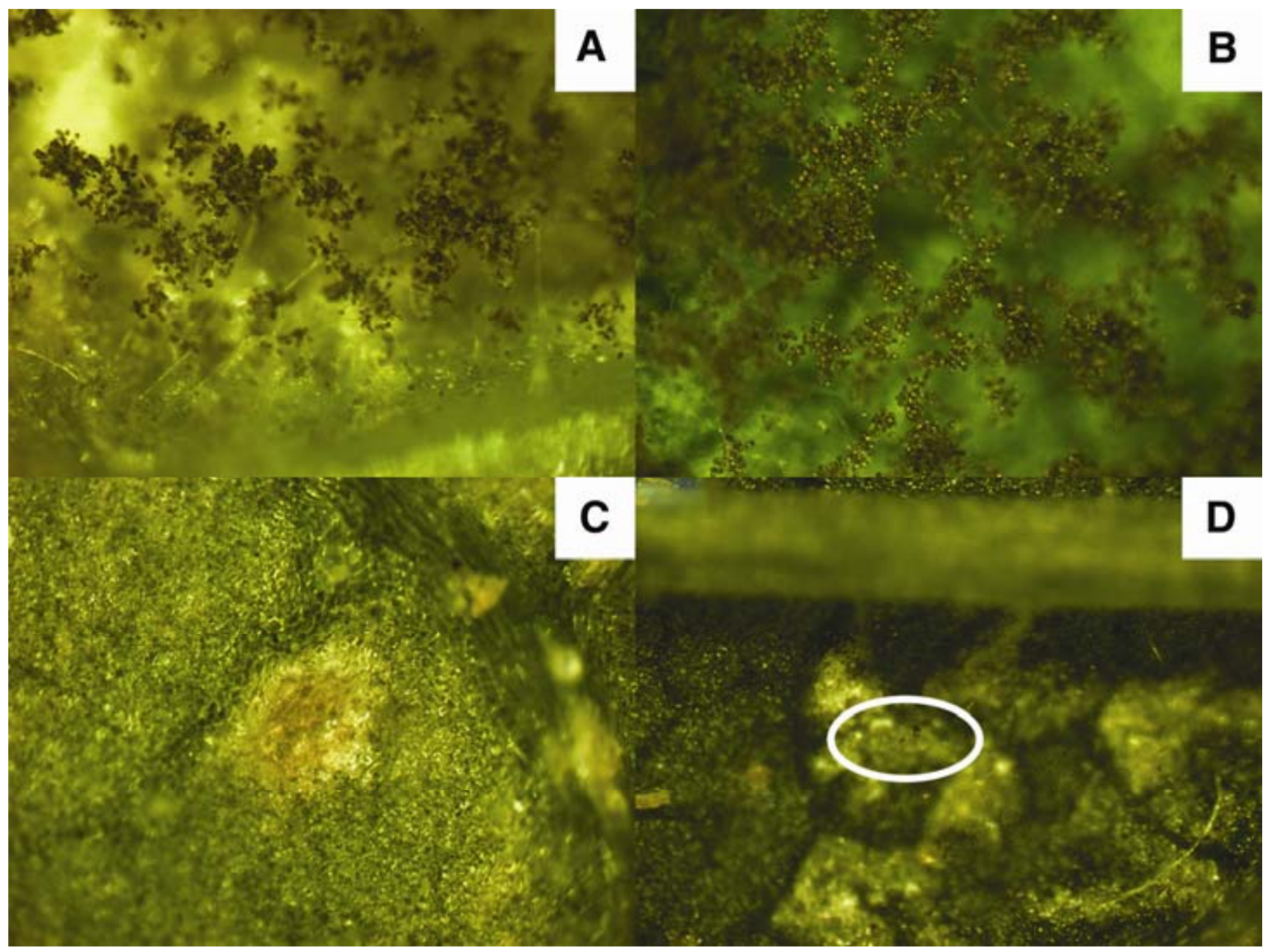

Fig. 5. Microscopic signs and symptoms from host-specificity experiments with Pseudoperonospora cubensis and $P$. humuli ( $\times 50$ magnification). A, P. cubensis inoculated on cucumber 'Straight 8'; B, P. humuli inoculated on hop 'Pacific Gem'; C, P. humuli inoculated on Straight 8; and D, P. cubensis inoculated on Pacific Gem. A and B, Positive controls are characterized by profuse sporulation and few hypersensitive-like lesions, whereas $\mathbf{C}$ and $\mathbf{D}$, the opposite is true of the reciprocal inoculations. Sporangia can be seen in the circled area of panel $\mathbf{D}$. 
recorded on at least 49 wild and cultivated species of Cucurbitaceae in 70 countries $(6,7,41)$. Voglmayr et al. (64) reported $P$. cubensis to be causing downy mildew on I. irvingii (family Balsaminaceae) in Cameroon, although pathogenicity studies were not reported on a cucurbit host with that isolate. We note again that the hop cultivars included in this study are highly susceptible to hop downy mildew and, under natural conditions, can be killed by $P$. humuli if rigorous disease management is not practiced. Thus, reduced level of sporulation of $P$. cubensis on these cultivars suggests that hop is not a primary or preferred host of this pathogen.

Because isolates of both $P$. cubensis and $P$. cubensis from Korea have phylogenetic histories divergent from other isolates from elsewhere in the world, host pathogenicity studies with these isolates, on both $H$. lupulus and $H$. japonicus, are needed to confirm their host range. Although $P$. humuli did not appear to be pathogenic on the reportedly universally susceptible cucumber and cantaloupe hosts utilized in the current study, experimenting with $P$. humuli on the differential set of cucurbit species of Lebeda and Gadasová (28) may reveal a host on which $P$. humuli is better able to colonize.

In addition to the differences found in this study, there are several biologically relevant differences in the life cycles of $P$. cubensis and $P$. humuli. Pathotypes (or race structures) of $P$. humuli have never been demonstrated, whereas there is evidence of the existence of a number of pathotypes of $P$. cubensis $(7,10$, $28,41,54)$. Oospores of $P$. cubensis are rarely found $(7,41)$. In contrast, oospores of $P$. humuli can be produced in large numbers in infected shoots, leaf lesions, and cones, although there is contradictory evidence of whether they are able to cause disease in the field $(1,3,4,8,9,31,43,48,55)$. Additionally, while $P$. humuli can overwinter as mycelia in perennating hop crowns and, potentially, as oospores in soil or plant debris (48), P. cubensis infects frost-sensitive plants and must survive the off-season in hosts living in warmer climates or greenhouses, on perennial weed hosts, or perhaps as oospores $(7,41,50)$.

In summary, phylogenetic and host-specificity analyses clearly indicate that there are biologically relevant characteristics that differentiate $P$. cubensis and $P$. humuli, with the exception of isolates from $H$. japonicus from Korea. Thus, the proposed synonymy (6) requires further consideration. Aside from two isolates of $P$. humuli on $H$. japonicus from Korea, phylogenetic analyses suggest that $P$. humuli belongs in a cluster separate from $P$. cubensis. These results are consistent with parallel research reported by Runge et al. (49), which also concluded that $P$. cubensis and $P$. humuli should not be considered conspecific. The results of our study suggest that reduction of $P$. humuli to a synonym of $P$. cubensis may be premature, particularly given the quantifiable differences in pathogenicity and genetic lineages between isolates from Humulus spp. and cucurbit hosts. Designation of a forma specialis is one means to differentiate important subtaxon differences in physiology (35), such as host range. From a molecular evolutionary perspective, forma specialis designations are not clearly understood and cannot be detected with sequence data. However, such designations are critical for preserving information on host range or preference and legal measures such quarantines.

The current study and emerging body of knowledge $(6,16$, 49,53 ) indicate that $P$. humuli and $P$. cubensis are morphologically and genetically very similar but possess measurable physiological

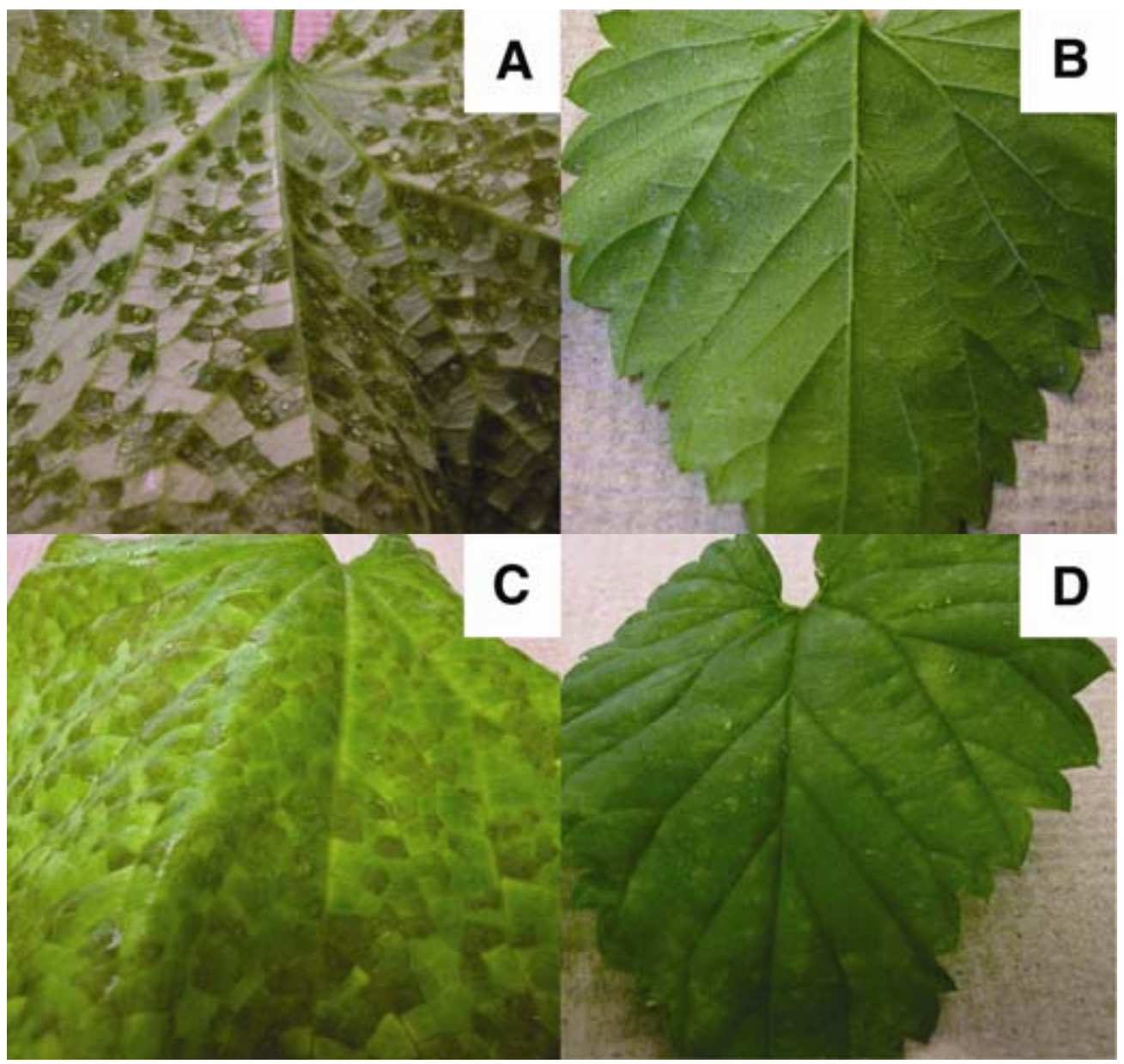

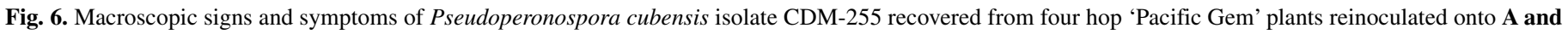

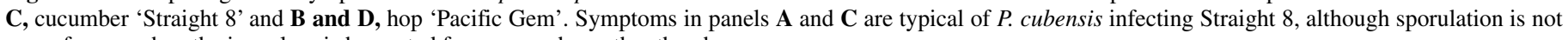
as profuse as when the inoculum is harvested from cucumber rather than hop. 
and genetic differences. Further investigations of the speciespopulation boundary, host specificity on $H$. japonicus and other hosts in Asia, and a more intensive sampling of Asian isolates of $P$. cubensis and $P$. humuli are needed to determine the true evolutionary history of this group. The true evolutionary history and species boundaries might be resolved by a coalescent analysis, as was done for Phytophthora ramorum (20). Until such data is available, we recommend retaining the two species names, P. cubensis and P. humuli.

\section{ACKNOWLEDGMENTS}

This work was financially supported by the United States Department of Agriculture-Agricultural Research Service CRIS 303-5358-22000040-00D, the Oregon Hop Commission, and the Oregon State University Agricultural Research Foundation. We thank G. Holmes and S. Colucci for providing isolates of $P$. cubensis as well as protocols for maintenance and preservation; K. Neufield, S. Koike, J. Patzak, and H.-D. Shin for providing live isolates or DNA that were vital to this research; N. Adair and J. Woods for excellent technical support and assistance; and S. Kousik, J. Woods, and the two anonymous reviewers and the senior editor for comments and suggestions that improved this article.

\section{LITERATURE CITED}

1. Arens, K. 1929. Untersuchungen über Pseudoperonospora humuli (Miyabe u. Takah.), den Erreger der neuen Hopfenkrankheit. Phytopathol. Z. 1:169-193.

2. Blair, J. E., Coffey, M. D., Park, S.-Y., Geiser, D. M., and Kang, S. 2008. A multi-locus phylogeny for Phytophthora utilizing markers derived from complete genome sequences. Fungal Genet. Biol. 45:266-277.

3. Bressman, E. M., and Nichols, A. A. 1933. Germination of the oospores of Pseudoperonospora humuli. Phytopathology 23:485-487.

4. Chee, H. Y., and Klein, R. E. 1998. Laboratory production of oospores in Pseudoperonospora humuli. Korean J. Plant Pathol. 14:618-621.

5. Chee, H. Y., Nelson, M. E., Grove, G. G., Eastwell, K. C., Kenny, S. T., and Klein, R. E. 2006. Population biology of Pseudoperonospora humuli in Oregon and Washington. Plant Dis. 90:1283-1286.

6. Choi, Y. J., Hong, S. B., and Shin, H. D. 2005. A re-consideration of Pseudoperonospora cubensis and $P$. humuli based on molecular and morphological data. Mycol. Res. 109:841-848.

7. Cohen, Y. 1981. Downy mildew of cucurbits. Pages 341-353 in: The Downy Mildews. D. M. Spencer, ed. Academic Press, New York.

8. Coley-Smith, J. R. 1962. Overwintering of hop downy mildew Pseudoperonospora humuli (Miy. \& Tak.) Wilson. Ann. Appl. Biol. 50:235-243.

9. Coley-Smith, J. R. 1965. Infection of hop rootstocks by downy mildew Pseudoperonospora humuli (Miy. \& Tak.) Wilson and its control by early season dusts. Ann. Appl. Biol. 56:381-388.

10. Colucci, S. J. 2008. Host Range, Fungicide Resistance and Management of Pseudoperonospora cubensis, Causal Agent of Cucurbit Downy Mildew. MS thesis, North Carolina State University, Raleigh.

11. Cooke, D. E. L., Drenth, A., Duncan, J. M., Wagels, G., and Brasier, C. M. 2000. A molecular phylogeny of Phytophthora and related oomycetes. Fungal Genet. Biol. 30:17-32.

12. Flier, W. G., Grünwald, N. J., Kroon, L. P. N. M., van den Bosch, T. B. M., Garay-Serrano, E., Lozoya Saldaña, H., Bonants, P. J. M., and Turkensteen, L. J. 2002. Phytophthora ipomoeae, a new homothallic species causing late blight on Ipomoeae longipedunculata in the Toluca Valley of central Mexico. Mycol. Res.106:848-856.

13. Gäumann, E. 1918. Über die Formen der Peronospora parasitica (Pers.) Fries. Ein Beitrag zur Speciesfrage bei den parasitischen Pilzen. Bot. Centralblatt. Beihefte 35:395-533.

14. Gent, D. H., Nelson, M. E., Farnsworth, J. L., and Grove, G. G. 2009. PCR detection of Pseudoperonospora humuli in air samples from hop yards. Plant Pathol. 58:1081-1091.

15. Gent, D. H., Nelson, M. E., and Grove, G. G. 2008. Persistence of phenylamide insensitivity in Pseudoperonospora humuli. Plant Dis. 92:463-468.

16. Göker, M., García-Blázquez, G., Voglmayr, H., Tellería, M. T., and Martín, M. P. 2009. Molecular taxonomy of phytopathogenic fungi: A case study in Peronospora. PLoS ONE 4:e6319.

17. Göker, M., Voglmayr, H., Riethmüller, A., and Oberwinkler, F. 2007. How do obligate parasites evolve? A multi-gene phylogenetic analysis of downy mildews. Fungal Genet. Biol. 44:105-122.

18. Göker, M., Voglmayr, H., Riethmüller, A, Weiß, M., and Oberwinkler, F. 2003. Taxonomic aspects of Peronosporaceae inferred from Bayesian molecular phylogenetics. Can. J. Bot. 81:672-683.
19. Goodwin, S. B., Legard, D. E., Smart, C. D., Levy, M., and Fry, W. E. 1999. Gene flow analysis of molecular markers confirms that Phytophthora mirabilis and P. infestans are separate species. Mycologia 91:796-810.

20. Goss, E. M., Carbone, I., and Grünwald, N. J. 2009. Ancient isolation and independent evolution of the three clonal lineages of the exotic sudden oak death pathogen Phytophthora ramorum. Mol. Ecol. 18:1161-1174.

21. Grünwald, N. J., and Flier, W. G. 2005. Biology of Phytophthora infestans at its center of origin. Annu. Rev. Phytopathol. 43:171-190.

22. Hall, G. S. 1996. Modern approaches to species concepts in downy mildews. Plant Pathol. 45:1009-1026.

23. Haunold, A., Likes, S. T., Nickerson, G. B., and Hampton, R. O. 1984. Registration of Nugget hop. Crop Sci. 24:618.

24. Hoerner, G. R. 1940. The infection capabilities of hop downy mildew. J. Agric. Res. 61:331-334.

25. Huelsenbeck, J. P., and Ronquist, F. 2001. MrBayes: Bayesian inference of phylogeny. Bioinformatics 17:754-755.

26. Kalloo, G., and Bergh, B. O. 1993. Genetic Improvement of Vegetable Crops. Pergamon Press, New York.

27. Kang, S., Mansfield, M. A., Park, B., Geiser, D. M., Ivors, K. L., Coffey, M. D., Grünwald, N. J., Martin, F. N., Lévesque, C. A., and Blair, J. E. 2010. The promise and pitfalls of sequence-based identification of plantpathogenic fungi and oomycetes. Phytopathology 100:732-737.

28. Lebeda, A, and Gadasová, V. 2002. Pathogenic variation of Pseudoperonospora cubensis in the Czech Republic and some other European countries. Acta Hortic. 588:137-141.

29. Lebeda, A., and Widrlechner, M. P. 2003. A set of Cucurbitaceae taxa for differentiation of Pseudoperonospora cubensis pathotypes. J. Plant Dis. Prot. 110:337-349.

30. Lévesque, C. A., and De Cock, A. W. A. M. 2004. Molecular phylogeny and taxonomy of the genus Pythium. Mycol. Res. 108:1363-1383.

31. Magie, R. O. 1942. The epidemiology and control of downy mildew on hops. Tech. Bull. N. Y. State Agric. Exp. Stn. 267:1-48.

32. Martin, F. N. 2000. Phylogenetic relationships among some Pythium species inferred from sequence analysis of the mitochondrially encoded cytochrome oxidase II gene. Mycologia 92:711-727.

33. Martin, F. N., and Tooley, P. W. 2003. Phylogenetic relationships among Phytophthora species inferred from sequence analysis of the mitochondrially-encoded cytochrome oxidase I and II genes. Mycologia 95:269284.

34. Martin, F. N., Tooley, P. W., and Blomquist, C. 2004. Molecular detection of Phytophthora ramorum, the causal agent of sudden oak death in California, and two additional species commonly recovered from diseased plant material. Phytopathology 94:621-631.

35. McNeill, J., Barrie, F. R., Burdet, H. M., Demoulin, V., Hawksworth, D. L., Marhold, K., Nicolson, D. H., Prado, J., Silva, P. C., Skog, J. E., Wiersema, J. H., and Turland, N. J., eds. 2006. International Code of Botanical Nomenclature (Vienna Code) Adopted by the Seventeenth International Botanical Congress, Vienna, Austria, July 2005. Koeltz, Königstein, Germany.

36. Miyabe, K., and Takahashi, Y. 1906. A new disease of the hop-vine caused by Peronoplasmopara humuli n. sp. Trans. Sapporo Nat. Hist. Soc. 1:149157.

37. Neve, R. A. 1991. Hops. Chapman and Hall, New York.

38. Nylander, J. A. A. 2004. MrModeltest v2. Program distributed by the author. Evolutionary Biology Centre, Uppsala University, Sweden.

39. Page, R. D. M. 1996. TreeView An application to display phylogenetic trees on personal computers. CABIOS 12:357-358.

40. Palti, J. 1974. The significance of pronounced divergences in the distribution of Pseudoperonospora cubensis on its crop hosts. Phytoparasitica 2:109-115.

41. Palti, J., and Cohen, Y. 1980. Downy mildew of cucurbits (Pseudoperonospora cubensis): The fungus and its hosts, distribution, epidemiology and control. Phytoparasitica 8:109-147.

42. Palti, J., and Kenneth, R. 1981. The distribution of downy mildew genera over the families and genera of higher plants. Pages 45-56 in: The Downy Mildews. D. M. Spencer, ed. Academic Press, New York.

43. Parker, T. 2007. Investigation of hop downy mildew through association mapping and observations of the oospore. Ph.D. dissertation, Oregon State University, Corvallis.

44. Peterson, A. B., and Rosendahl, S. 2000. Phylogeny of the Peronosporomycetes (Oomycota) based on partial sequences of the large ribosomal subunit (LSU rDNA). Mycol. Res. 104:1295-1303.

45. Riethmüller, A., Voglmayr, H., Göker, M., Weiß, M., and Oberwinkler, F. 2002. Phylogenetic relationships of the downy mildews (Peronosporales) and related groups based on nuclear large subunit ribosomal DNA sequences. Mycologia 94:834-849.

46. Robinson, R. W., and Decker-Walters, D. S. 1997. Cucurbits. CAB International, New York.

47. Ronquist, F., and Huelsenbeck, J. P. 2003. MrBayes 3: Bayesian phylo- 
genetic inference under mixed models. Bioinformatics 19:1572-1574.

48. Royle, D. J., and Kremheller, H. T. 1981. Downy mildew of the hop. Pages 395-419 in: The Downy Mildews. D. M. Spencer, ed. Academic Press, New York.

49. Runge, F., Choi, Y. J., and Thines, M. 2011. Phylogenetic investigations in the genus Pseudoperonospora reveal overlooked species and cryptic diversity in the P. cubensis species cluster. Eur. J. Plant Pathol. 129:135146.

50. Runge, F., and Thines, M. 2009. A potential perennial host for Pseudoperonospora cubensis in temperate regions. Eur. J. Plant Pathol. 123:483486.

51. Runge, F., and Thines, M. 2011. Host matrix has major impact on the morphology of Pseudoperonospora cubensis. Eur. J. Plant Pathol. 129:147-156.

52. Salmon, E. S., and Ware, W. M. 1928. Inoculation experiments with the downy mildews of the hop and nettle (Pseudoperonospora humuli (Miy. et Taka.) Wils. and P. urticae (Lib.) Salmon et Ware). Ann. Appl. Biol. 15:352-370.

53. Sarris, P., Abdelhalim, M., Kitner, M., Skandalis, N., Panopoulos, N., Doulis, A., and Lebeda, A. 2009. Molecular polymorphisms between populations of Pseudoperonospora cubensis from Greece and the Czech Republic and the phytopathological and phylogenetic implications. Plant Pathol. 58:933-943.

54. Shetty, N. V., Wehner, T. C., Thomas, C. E., Doruchowski, R. W., and Shetty, K. P. V. 2002. Evidence for downy mildew races in cucumber tested in Asia, Europe, and North America. Sci. Hortic. 94:231-239.

55. Skotland, C. B. 1961. Infection of hop crowns and roots by Pseudoperonospora humuli and its relation to crown and root rot and overwintering of the pathogen. Phytopathology 51:241-244.

56. Stamatakis, A. 2006. RAxML-VI-HPC: Maximum likelihood-based phylogenetic analyses with thousands of taxa and mixed models. Bioinformatics 22:2688-2690.

57. Stamatakis, A., Hoover, P., and Rougemont, J. 2008. A fast bootstrapping algorithm for the RAxML web-servers. Syst. Biol. 57:758-771.

58. Swofford, D. L. 2003. PAUP*. Phylogenetic Analysis Using Parsimony (*and Other Methods). Version 4. Sinauer Associates, Sunderland, MA.

59. Thines, M., Voglmayr, H., and Göker, M. 2009. Taxonomy and phylogeny of the downy mildews (Peronosporaceae). Pages 47-75 in: Oomycete Genetics and Genomics. K. Lamour and S. Kamoun, eds. John Wiley \& Sons, Inc., Hoboken, NJ.

60. Thomas, C. E. Inaba, T., and Cohen, Y. 1987. Physiological specialization in Pseudoperonospora cubensis. Phytopathology 77:1621-1624.

61. Thompson, J. D., Higgins, D. G., and Gibson, T. J. 1994. CLUSTAL W: Improving the sensitivity of progressive multiple sequence alignment through sequence weighting, position specific gap penalties and weight matrix choice. Nucleic Acids Res. 22:4673-4680.

62. Voglmayr, H. 2003. Phylogenetic relationships of Peronospora and related genera based on nuclear ribosomal ITS sequences. Mycol. Res. 107:1132-1142.

63. Voglmayr, H. 2008. Progress and challenges in systematic of downy mildews and white blister rusts: New insights from genes and morphology. Eur. J. Plant Pathol. 122:3-18.

64. Voglmayr, H., Piatek, M., and Mossebo, D. C. 2009. Pseudoperonospora cubensis causing downy mildew disease on Impatiens irvingii in Cameroon: A new host for the pathogen. Plant Pathol. 58:394.

65. White, T. J., Bruns, T., Lee, S., and Taylor, J. 1990. Amplification and direct sequencing of fungal ribosomal RNA genes for phylogenetics. Pages 29-50 in: PCR Protocols. A Guide to Methods and Applications. M. A. Innis, D. H. Gelfand, J. J. Sninsky, and T. J. White, eds. Academic Press, San Diego, CA. 